\title{
A 3D view of the Hyades stellar and sub-stellar population ${ }^{\star}$
}

\author{
N. Lodieu ${ }^{1,2,3}$, R. L. Smart ${ }^{3}$, A. Pérez-Garrido ${ }^{4}$, and R. Silvotti ${ }^{3}$ \\ 1 Instituto de Astrofísica de Canarias (IAC), Calle Vía Láctea s/n, 38200 La Laguna, Tenerife, Spain \\ e-mail: nlodieu@iac.es \\ 2 Departamento de Astrofísica, Universidad de La Laguna (ULL), 38206 La Laguna, Tenerife, Spain \\ 3 Istituto Nazionale di Astrofisica, Osservatorio Astrofisico di Torino,Strada Osservatorio 20, 10025 Pino Torinese, Italy \\ ${ }^{4}$ Dpto. Física Aplicada, Universidad Politécnica de Cartagena, 30202 Cartagena, Murcia, Spain
}

Received 8 August 2018 / Accepted 14 January 2019

\begin{abstract}
Aims. Our scientific goal is to provide a 3D map of the nearest open cluster to the Sun, the Hyades, combining the recent release of Gaia astrometric data, ground-based parallaxes of sub-stellar member candidates and photometric data from surveys which cover large areas of the cluster.

Methods. We combined the second Gaia release with ground-based $H$-band parallaxes obtained with the infrared camera on the $2 \mathrm{~m}$ robotic Liverpool telescope to astrometrically identify stellar and sub-stellar members of the Hyades, the nearest open cluster to the Sun.

Results. We find 1764 objects within $70^{\circ}$ radius from the cluster centre from the Gaia second data release, whose kinematic properties are consistent with the Hyades. We limit our study to $30 \mathrm{pc}$ from the cluster centre $(47.03 \pm 0.20 \mathrm{pc})$ where we identify 710 candidate members, including 85 and 385 in the core and tidal radius, respectively. We determine proper motions and parallaxes of eight candidate brown dwarf members and confirm their membership. Using the 3D positions and a model-based mass-luminosity relation we derive a luminosity and mass function in the $0.04-2.5 M_{\odot}$ range. We confirm evidence for mass segregation in the Hyades and find a dearth of brown dwarfs in the core of the cluster. From the white dwarf members we estimate an age of $640_{-49}^{+67} \mathrm{Myr}$.

Conclusions. We identify a list of members in the Hyades cluster from the most massive stars down to the brown dwarfs. We produce for the first time a 3D map of the Hyades cluster in the stellar and sub-stellar regimes and make available the list of candidate members.
\end{abstract}

Key words. stars: low-mass - brown dwarfs - surveys - open clusters and associations: individual: Hyades - astrometry

\section{Introduction}

The large majority of stars are born in groups, clusters, and/or associations rather than in isolation (Lada \& Lada 2003). Investigating the dynamics of clusters is key to understanding their formation and subsequent evolution. Up to now, ground-based surveys mainly provided proper motion information as well as partial radial velocity measurements offering a two-dimensional map of the sky and, in particular for this work, of nearby open clusters. Using accurate astrometric observations of the Hipparcos satellite (High precision parallax collecting satellite; Perryman et al. 1997), a catalogue of about 120000 stars brighter than visual magnitude $V=12.4$ mag with a completeness limit of $V \sim 8 \mathrm{mag}$ was generated. Later, the Tycho-2 catalogue was created containing 2.5 million stars down to $V=11.5$ and $99 \%$ complete to $V \sim 10.5$ mag over the full sky (Høg et al. 2000). The advent of Gaia (Gaia Collaboration 2016) is opening an unprecedented window with accurate proper motions and parallaxes for more than one billion stars down to $G \sim 20.7$ mag, allowing a spatial investigation of our Galaxy at large radii never before investigated to that level of precision. In particular, Gaia provides accurate 3D space motions for the nearest and youngest open clusters to the Sun, including the Hyades.

The Hyades cluster (M25, Collinder 50, Caldwell 41) is the closest stellar cluster to the Sun. Using the Hipparcos

\footnotetext{
* Full Table C.1 is only available at the CDS via anonymous ftp to cdsarc.u-strasbg. fr (130.79.128.5) or via http://cdsarc. u-strasbg.fr/viz-bin/qcat?J/A+A/623/A35
}

catalogue Perryman et al. (1998) derived a mean distance of $46.34 \pm 0.27 \mathrm{pc}$ and a proper motion in the $74-140 \mathrm{mas} \mathrm{yr}^{-1}$ range. The core radius of the cluster is approximately $2.5-3.0 \mathrm{pc}$ while its tidal radius is about $10 \mathrm{pc}$ (Perryman et al. 1998; Röser et al. 2011). The reddening towards the cluster is negligible $(E(B-V) \leq 0.001 \mathrm{mag}$; Taylor 2006a).

The age of the cluster has been estimated with different methods, yielding a canonical age of $650 \pm 100 \mathrm{Myr}$. Comparison of the observed cluster sequence with model isochrones which include convective overshooting suggests $625 \pm 50$ Myr while evolutionary models (Maeder \& Mermilliod 1981; Mazzei \& Pigatto 1988; Mermilliod 1981) with enhanced convective overshooting give much older ages up to $1.2 \mathrm{Gyr}$ (Mazzei \& Pigatto 1988) that cannot be discarded (Eggen 1998; Tremblay et al. 2012). The ages determined from the cooling age of white dwarf members is $648 \pm 45 \mathrm{Myr}$ (De Gennaro et al. 2009) while stellar binaries suggest $\sim 650 \mathrm{Myr}$ (Lebreton et al. 2001). The role of rotation at such ages leads to an age of $750 \pm 100$ Myr (Brandt \& Huang 2015). Recently, the method using the lithium depletion boundary at the stellar to substellar limit prompted an age of $650 \pm 70 \mathrm{Myr}$ consistent with the canonical age of the cluster (Lodieu et al. 2018; Martín et al. 2018). The metallicity has been subject to debate with discrepant estimates suggesting a mean metallicity close to solar $(\mathrm{Fe} / \mathrm{H}=0.05 \pm 0.05$; Gebran et al. 2010) or slightly super-solar between $0.127 \pm 0.022 \mathrm{dex}$ and $0.14 \pm 0.10 \mathrm{dex}$ (Boesgaard \& Friel 1990; Cayrel de Strobel et al. 1997; Grenon 2000). 
The surveys targeting the Hyades can be divided up into two main groups: the large-scale studies looking for a complete census of the cluster members over very large areas of the sky (Gizis et al. 1999; Goldman et al. 2013; Hanson 1975; Hogan et al. 2008; Reid 1992; Röser et al. 2011) or deeper surveys on small(er) patches in the cluster centre to identify new members (Bouvier et al. 2008; Dobbie et al. 2002; Hanson 1975; Leggett et al. 1994; Melnikov \& Eislöffel 2018; Reid \& Gizis 1997; Reid et al. 1999; Reid 1993; Stauffer et al. 1995, 1994). Subsequent spectroscopic follow-up has been conducted for many sources to confirm their membership via spectral typing, radial velocity and/or lithium content (Bryja et al. 1994; Leggett \& Hawkins 1989; Mermilliod et al. 2009; Reid \& Hawley 1999; Reid \& Mahoney 2000; Soderblom et al. 1995; Stauffer et al. 1995, 1994; Tabernero et al.2012; White et al. 2007). The current census down to approximately $0.1 M_{\odot}$ is summarised in Röser et al. (2011) using the Positions and Proper Motion Extra Large catalogue (PPMXL; Röser et al. 2010) and the Panoramic Survey Telescope and Rapid Response System first data release (Pan-STARRS DR1; Chambers et al. 2016; Goldman et al. 2013; Kaiser et al. 2002) complemented by the L and T dwarf member candidates analysed by Hogan et al. (2008) and Bouvier et al. (2008), respectively. The coolest members have been confirmed spectroscopically with masses below the hydrogen-burning limit (Bouvier et al. 2008; Casewell et al. 2014; Lodieu et al. 2014a, 2018; Martín et al. 2018).

In this manuscript, we present an astrometric selection of Hyades cluster member candidates from the second Gaia data release (Gaia Collaboration 2018b) yielding a revised census and a 3D map with positions in the sky. In Sect. 2 we present the input catalogue used for our study of the Hyades cluster and compile a list of previously-known members in Sect. 3. In Sect. 4 we describe complementary ground-based parallaxes from a dedicated programme carried out with the Liverpool telescope for the coolest member candidates of the cluster. In Sect. 5 we identify member candidates in the Hyades from Gaia DR2 and compare with previous studies to address the completeness and contamination of our sample. We dedicate Sect. 6 to the analysis of white dwarf members and their implication on the age of the cluster that we compare with other estimates. In Sect. 7 we derive the luminosity and mass functions in the stellar and sub-stellar regimes. In Sect. 8 we discuss the spatial distribution of the highest probability member candidates and present the first 3D map of the Hyades from the most massive members down to the sub-stellar regime.

\section{The Gaia DR2 sample}

We made use of the Gaia DR2 data (Gaia Collaboration 2016, 2018b) released world-wide on April 25th 2018. Our objective was to start as inclusive as possible and to be more selective later in the process. Initially we started with all Gaia DR2 objects that were within $70^{\circ}$ of the nominal cluster centre at $\alpha=67.0^{\circ}$, $\delta=+16.0^{\circ}$ and had a parallax greater the 10 mas, that is within $100 \mathrm{pc}$, which results in 126144 objects (Fig. 1). We believe that the Hyades cluster has a on sky dimension of less than $30 \mathrm{pc}$ (Röser et al. 2011). By extending the search from 30 to $70^{\circ}$, our analysis shall include all possible members with measured parallaxes but we will restrain our analysis to an volume of $30 \mathrm{pc}$ from the cluster centre.

We cross-matched this catalogue with a series of wellknown large-scale surveys to provide photometry over a wide wavelength range, keeping all Gaia DR2 sources without any

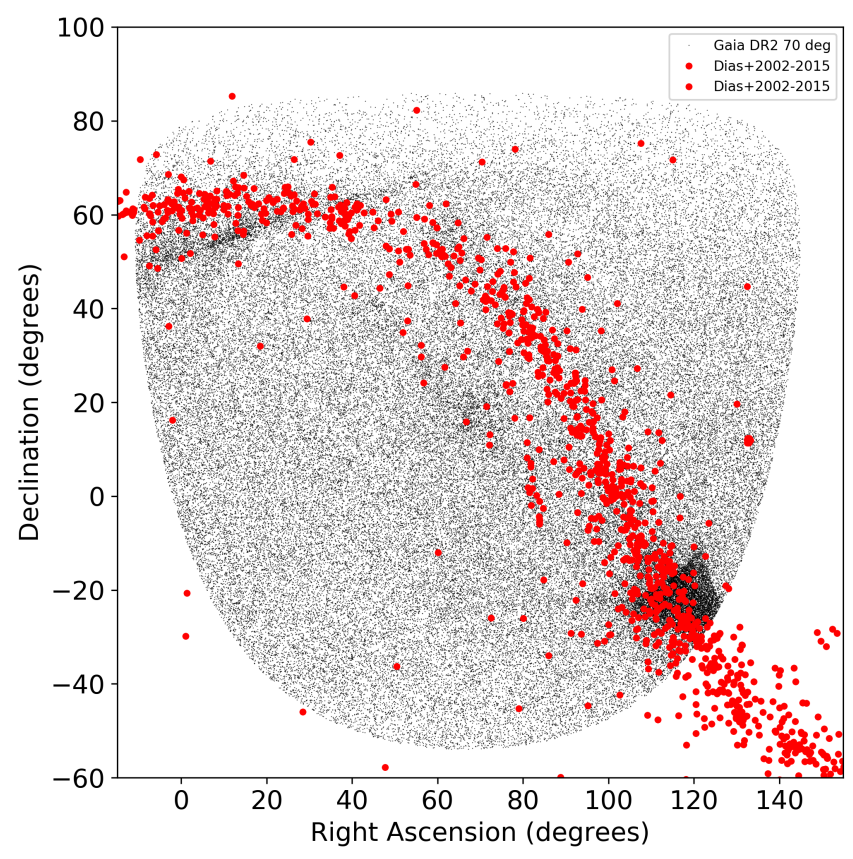

Fig. 1. Distribution of all sources in a radius of $70^{\circ}$ from the cluster centre (small black dots) along with open clusters close to galactic plane (Dias et al. 2002, 2006, 2014, 2018).

counterpart in those surveys either due to coverage or brightnessfaintness reasons. The most precise match was obtained with a maximum distance of $10^{\prime \prime}$. We did not use the official Gaia DR2 cross-match tables but our own script with stilts (Taylor 2006b) because not all public large-scale catalogues are available with the online archive. All the unresolved sources in the large-scale surveys that were resolved in Gaia were matched to the closest Gaia source which is normally the brighter of the two possible matches. All matches were made at the epoch of the target catalogues by applying the Gaia proper motions to possible counterparts. The number of matches within $10^{\prime \prime}$ are 103317 objects from the Two Micron All-Sky Survey (hereafter 2MASS; Cutri et al. 2003; Skrutskie et al. 2006), 38889 from the Sloan Digital Sky Survey data release 12 (SDSS DR12; Abolfathi et al. 2018); 6775 from the UKIRT Infrared Deep Sky Survey Galactic Clusters Survey (UKIDSS GCS; Lawrence et al. 2007); 107009 from the Wide-field Infrared Survey Explorer (AllWISE; Wright et al. 2010; Cutri et al. 2014); and 118144 from the first data release of the Pan-STARRS DR1 (PS1; Kaiser et al. 2002; Chambers et al. 2016). All surveys completely covered the total region apart from UKIDSS which only covered $\sim 20$ square degres centred on the Hyades nominal centre.

\section{Previously known members}

\subsection{Compilation of known members}

We compiled a list of known members from different surveys published over the past decade which represent the most complete surveys in the Hyades. We started with the following sub-samples: 724 stars from Röser et al. (2011), 773 stars from Goldman et al. (2013) as well as 20 candidates from Dobbie et al. (2002). We also included ten confirmed L dwarfs from Hogan et al. (2008) confirmed spectroscopically by Casewell et al. (2014) and Lodieu et al. (2014b), two mid-L dwarfs from Schneider et al. (2017), one L5 from Pérez-Garrido et al. (2017), and the two 
N. Lodieu et al.: A 3D view of the Hyades stellar and sub-stellar population

Table 1. Hyades late-M and early-L confirmed spectroscopically with Gaia proper motions and distances.

\begin{tabular}{|c|c|c|c|c|c|c|c|c|}
\hline Name & $\begin{array}{c}\text { RA } \\
\text { (hh:mm:ss.ss) } \\
\end{array}$ & $\begin{array}{c}\text { Dec } \\
\text { (dd:":".") }\end{array}$ & $\mathrm{SpT}$ & SourceID & $\begin{array}{c}\text { Plx } \\
\text { (mas) }\end{array}$ & $\begin{array}{c}\text { pmRA } \\
\left(\operatorname{mas~yr}^{-1}\right)\end{array}$ & $\begin{array}{c}\mathrm{pmDEC} \\
\left(\mathrm{mas} \mathrm{yr}^{-1}\right) \\
\end{array}$ & $\begin{array}{c}H \\
(\mathrm{mag})\end{array}$ \\
\hline Hya & 0 & 0 & & 8 & 5 & 76 & 50 & 13.85 \\
\hline Hyal & & & $\mathrm{LC}$ & 72 & $183 \pm$ & 107.981 & -11.185 & 14.78 \\
\hline Hya04 & 59 & 37.3 & M9.5 & 3409343115420601728 & - & - & - & 14.97 \\
\hline Hya06 & $: 22: 05.22$ & $+13: 58: 47.3$ & M9.5 & 3310992904122021120 & $18.2032 \pm 0.9181$ & $89.391 \pm 1.612$ & $-17.657 \pm 1.307$ & 14.81 \\
\hline Hya08 & 04:58:45.75 & $+12: 12: 34.1$ & L0.5 & 3295377360811741184 & $24.2448 \pm 0.9774$ & $85.748 \pm 2.536$ & $-16.008 \pm 1.265$ & 14.55 \\
\hline
\end{tabular}

Notes. Hya05 (M3) and Hya07 (M5) are not listed below because they were rejected as spectroscopic members of the Hyades based on their optical spectra (Lodieu et al. 2014b). The other L dwarfs are not in Gaia but listed in Table 2 with ground-based parallaxes.

T dwarfs from Bouvier et al. (2008). After removing common sources from these catalogues, we are left with 837 highprobability candidates with proper motion and photometry consistent with membership to the Hyades.

\subsection{Cross-correlation with Gaia DR2}

We cross-matched this list with Gaia DR2 with a matching radius of $3^{\prime \prime}$, returning 825 sources equivalent to a recovery rate of $99 \%$. The sources not recovered are mainly substellar, including HyaL5 (Pérez-Garrido et al. 2017), the two mid-L dwarfs from Schneider et al. (2017), the two T dwarfs from Bouvier et al. (2008), Hya09 and Hya12 from Hogan et al. (2008), as well as four sources (ID =30, 324, 651, 671) from Goldman et al. (2013) and one (h7334b) from Dobbie et al. (2002). The ten brightest Hyades members are also recorded in the Gaia DR2 catalogue. We conclude that Gaia is complete down to the hydrogen-burning limit but incomplete in the sub-stellar regime in the Hyades cluster because it is missing the seven aforementioned brown dwarfs. We do not know why the five targets above from Goldman et al. (2013) and Dobbie et al. (2002) are missing from DR2 (less than 1\%). We checked that those objects are real on the Digital Sky Survey and 2MASS images although one of them (h7334b) appears faint, which may explain why it has no entry in Gaia. Three of them have proper motions quoted in Simbad and two of them (Goldman30, Goldman324) have entries in the Gaia DR2 catalogue without astrometry but effective temperature estimates and 2-parameter solutions. We also note that Goldman30 is classified as a M5 at 22 pc by Newton et al. (2015) and Goldman671 might belong to a young moving group (Gagné et al. 2015a).

We cross-correlated these 837 pre-Gaia members with our catalogue within $70^{\circ}$ from the Hyades centre and recovered 749 of these previously-known sources. The objects not recovered in our catalogue $(837-749)$ have parallaxes less than 10 mas, which is our lower limit to create the $70^{\circ}$ catalogue.

We have also cross-correlated this list of 749 pre-Gaia members with the catalogue of 515 Hyades sources published by Gaia Collaboration (2018a), yielding 415 objects in common. We used the SourceID parameter for the cross-correlation to avoid any mis-matching based on coordinates. Therefore, we conclude that earlier surveys of the Hyades may have excluded about $20 \%$ of known members if the new candidates from Gaia Collaboration (2018a) are confirmed as members. We note that Röser et al. (2011) estimated a level of contamination of the order of $\sim 9 \%$ with 65 field stars in their sample of 724 Hyades candidates based on a control sample of stars with HipParcos parallaxes and/or published radial velocities.

\section{Ground-based parallaxes of Hyades L/T dwarfs}

\subsection{Targets}

We selected the faintest Hyades members confirmed spectroscopically from three surveys. First, ten of the 12 ultracool dwarf candidates from Hogan et al. (2008), confirmed spectroscopically as late-M and early-L dwarfs by Casewell et al. (2014) and Lodieu et al. (2014b). Second, the recent L5 dwarf discovered by Pérez-Garrido et al. (2017) and confirmed as a sub-stellar member by Lodieu et al. (2018). Third, the two early-T dwarf members reported by Bouvier et al. (2008), the coolest members known to date.

We estimated the Gaia $G$-band magnitude of these sources with the equation $G-J \sim 0.244 \times \mathrm{SpT}-12.633$, where $\mathrm{SpT}$ is the numerical counterpart of the spectral type with $\mathrm{L} 0 \equiv 70$ and $\mathrm{T} 2 \equiv 82$ as examples (Smart et al. 2017a). We discarded the confirmed late-M and $\mathrm{L}$ dwarfs brighter than $G=20.7 \mathrm{mag}$ because we predicted they would have Gaia astrometry. We programmed the remaining eight sources for astrometric followup with the infrared camera on the Liverpool telescope. This strategy has been proven to be effective because all the sources not included in our parallax programme have Gaia distances (Table 1), except Hya04 (2MASS J04421859+1754373; Hogan et al. 2008) which has been included in the Gaia DR2 $(\mathrm{ID}=3409343115420601728)$ with just positions; we expect it to have full astrometry in the next release.

\subsection{Observations}

We targeted the eight Hyades $\mathrm{L}$ and $\mathrm{T}$ dwarf member candidates with the infrared camera IO:I (Barnsley et al. 2016) on the 2-m robotic Liverpool telescope (Steele et al. 2004) over three semesters between August 2015 and January 2018 (CL15B06, CL16B03, CL17B01; PI Lodieu). We requested a seeing better than $1.5^{\prime \prime}$ and an elevation on the sky higher than $30^{\circ}$. We also asked for a sky brightness better than the "dark+4 magnitudes" definition of the Liverpool telescope, which means that our programme could be observed with bright moon and astronomical twilight in the worst case.

The IO:I instrument was installed on the Liverpool telescope in August 2015. It uses a $2048 \times 2048$ pixel Hawaii 2 RG offering a field of view of 6.3 arcmin and a pixel scale of $0.18^{\prime \prime}$. It is currently solely equipped with a $H$-band filter. The blue and red cut-offs at 1.4 and 1.8 microns are set by the detector and the filter, respectively.

To optimise the determination of the parallax from the ground, we designed the following strategy. We requested seven observations per semester for each target, distributed as follows: three points during morning and evening twilights spread over 1.5 months and separated by about two weeks to maximise the 

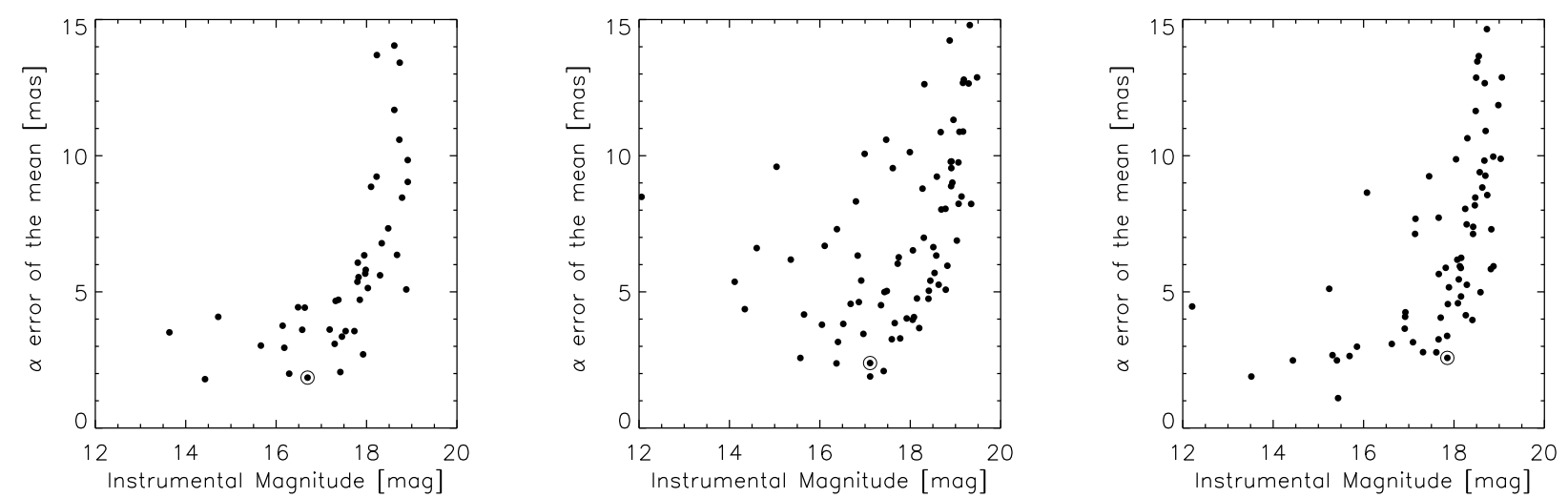

Fig. 2. Root-mean-square of the error of the mean between the first IO:I epoch and the remaining epochs as a function of instrumental $H$-band magnitude for three of the eight targets (Hya02, Hya09, and HyaL5 from left to right) for which we determined ground-based distances. The circled objects are the targets.

parallax factors and an additional point in the middle of the night to improve the proper motion solution. We repeated this strategy for three semesters over three years. However, the past two winter semesters have suffered from significantly poor weather, yielding a small number of points during the past two years. Nonetheless, we were able to collect between ten and 17 points per object (Table A.1-A.8).

We employed the same dithering procedure for all targets: we used individual on-source integrations of $10 \mathrm{~s}$ with a 9-point dither pattern to optimise the sky subtraction in the $H$-band. We repeated this sequence seven times for all objects, yielding a total on-source exposure time of $630 \mathrm{~s}$, except for the two faintest targets: the L5 dwarf (Pérez-Garrido et al. 2017) and CFHT-Hy-20 (Bouvier et al. 2008), which we repeated ten times for a total time of $900 \mathrm{~s}$.

\subsection{Data reduction}

We downloaded directly from the archive the reduced images from the automatic pipeline designed for IO:I (Barnsley et al. 2016). We refer the reader to that paper for more detailed information. To summarise, the pipeline includes bias subtraction, non-linearity correction, flat fielding, bad pixel masking, and sky subtraction for each of the 9-point dither position resulting in seven or ten repeated images with a world coordinate system incorporated.

In a second step, we combined the seven or ten repeated positions with the imcombine task under IRAF (Tody 1986, 1993) to create a final image for each target equivalent to the total onsource integrations quoted in Sect. 4.2. The stacking and offsetting of the individual repeats were performed within the task imcombine using the offsets from the header. We verified that the full-width-half-maximum on the final image was consistent with the range of seeing measured on individual repeats. We used the combined images to proceed with the centroiding procedure to derive trigonometric parallaxes (Fig. 2). We carried out the centroiding of all objects in the combined images using the Cambridge Astronomy Survey Unit's imcore maximum likelihood barycentre (CASUTOOLS; v 1.0.21 ${ }^{1}$ ). We also tried other packages but the centroiding from this package was the most robust and provided the most consistent floor to the precision as shown in Fig. 2.

\footnotetext{
casu.ast. cam.ac.uk
}

\subsection{Astrometric parameter determination}

The astrometric reduction was carried out using the Torino Observatory Parallax programme pipeline procedures and the reader is referred to Smart et al. (2003) for details and Smart et al. (2010) for some results. Here we just outline the main steps of the procedure. A base frame, observed on a night with good seeing, was selected and the measured $\mathrm{x}, \mathrm{y}$ positions of all objects were transformed to a standard coordinate $\xi, \eta$ system determined from a gnomic projection of the Gaia DR2 objects in the frame. All subsequent frames were transformed to this standard coordinate system with a simple six constant linear astrometric fit using all common objects except the target. We then removed any frames that had an average reference star error larger than three times the mean error for all frames in either coordinate, or, had less than 12 stars in common with the base frame. This cleaning resulted in one frame being removed in the solutions of Hya10/Hya12/HyaL5 out of 12/17/12 frames, respectively, and two frames from the 15 in the Hya19 sequence.

Since the target is not used in the fit, its positional change is a reflection of its parallax and proper motion. We fit a simple five parameter model to this positional change, and that of all the other objects in the field, to find their astrometric parameters implicitly assuming that all objects are single. We then iterate this procedure where, in addition to removing frames as described above, we also remove objects with large errors over the sequence from the sub-set used to astrometrically align frames. Finally, for the target we removed any observations where the combined residuals of the two coordinates is greater than three times the sigma of the whole solution. No attempt was made to improve the astrometric fit by assuming a binary system because the length of the observational sequence and small number of observations would not support such a fit.

The solutions were tested for robustness using bootstrap-like testing where we iterate through the sequence selecting different frames as the base frame thus computing many solutions that incorporate varied sets of reference stars and starting from different dates. We create the sub-set of all solutions with: (i) a parallax within $1 \sigma$ of the median solution; (ii) the number of included observations in the top 10\%; and (iii) at least 12 reference stars in common to all frames. From this sub-set, for this publication, we have selected the one with the smallest error. More than $90 \%$ of the solutions were within $1 \sigma$ of the published solution.

To the relative parallaxes we add a correction to find astrophysically useful absolute parallaxes. This correction is estimated from 
Table 2. Hyades L/T members with ground-based parallaxes from the Liverpool telescope.

\begin{tabular}{|c|c|c|c|c|c|c|c|c|c|c|}
\hline Name & $\begin{array}{c}\alpha(2015.5) \\
\text { (hh:mm:ss.sss) }\end{array}$ & $\begin{array}{c}\delta(2015.5) \\
\text { (dd:mm:ss.sss) }\end{array}$ & $\mathrm{SpT}$ & $\begin{array}{c}\text { Baseframe } \\
\text { (year) }\end{array}$ & $N_{\text {obs }}, N_{\text {star }}$ & $\begin{array}{c}\varpi_{\mathrm{abs}} \\
(\mathrm{mas})\end{array}$ & $\begin{array}{l}\text { COR } \\
\text { (mas) }\end{array}$ & $\begin{array}{c}\mu_{\alpha} \cos \delta \\
\left(\operatorname{mas~yr}^{-1}\right)\end{array}$ & $\begin{array}{c}\mu_{\delta} \\
\left(\operatorname{mas~yr}^{-1}\right)\end{array}$ & $\begin{array}{l}\Delta \mathrm{T} \\
(\mathrm{yr})\end{array}$ \\
\hline Hya02 & $03: 52: 46.433$ & $+21: 12: 32.805$ & $\mathrm{~L} 1.5$ & 2016. & 11,36 & $17.7 \pm$ & 0.96 & 116. & $-26.9 \pm 1.5$ & 2.38 \\
\hline Hya10 & $04: 17: 33.988$ & $+14: 30: 15.360$ & $\mathrm{~L} 2.0$ & 2015.7622 & 36 & $28.5 \pm$ & 0.96 & $120.2 \pm$ & -12.2 & 2.39 \\
\hline Hya11 & $03: 55: 42.143$ & $+22: 57: 01.073$ & L3.5 & 2016.7371 & 10,47 & $39.1 \pm 16.3$ & 0.96 & $138.1 \pm 13.3$ & $-19.4 \pm 9.0$ & 2.37 \\
\hline Hya12 & $04: 35: 43.043$ & $+13: 23: 44.877$ & L3.0 & 2016.8054 & 16,63 & $24.1 \pm 2.1$ & 0.92 & $100.2 \pm 1.9$ & $-15.1 \pm 2.0$ & 2.33 \\
\hline Hya19 & $04: 46: 35.444$ & $+14: 51: 25.951$ & L4.0 & 2016.7342 & 13,80 & $20.6 \pm 2.5$ & 0.82 & $76.3 \pm 2.9$ & $-17.7 \pm 1.5$ & 2.12 \\
\hline HyaL5 & $04: 18: 35.011$ & $+21: 31: 26.788$ & L5.0 & 2016.7315 & 11,67 & $25.8 \pm 2.9$ & 0.81 & $141.5 \pm 2.7$ & $-45.7 \pm 2.3$ & 2.35 \\
\hline CFHT-Hy-20 & $04: 30: 38.887$ & $+13: 09: 56.636$ & $\mathrm{~T} 2.0$ & 2018.0531 & 13,48 & $30.8 \pm 3.0$ & 0.77 & $141.3 \pm 2.9$ & $-14.5 \pm 3.2$ & 2.37 \\
\hline CFHT-Hy-21 & 04:29:22.869 & $+15: 35: 29.842$ & $\mathrm{~T} 1.0$ & 2015.7815 & 10,72 & $33.5 \pm 12.7$ & 0.79 & $82.1 \pm 9.8$ & $-15.5 \pm 8.6$ & 2.38 \\
\hline
\end{tabular}

Notes. We give the names, coordinates at epoch 2015.5 to be consistent with Gaia DR2 (Table 1), optical spectral types (Lodieu et al. 2014b; Martín et al. 2018), epochs of the base frame, the number of observations used in the solutions $\left(N_{\text {obs }}\right)$ with the numbers of reference stars entering the fit $\left(N_{\text {star }}\right)$, the corrections from relative to absolute parallax (COR), the proper motions, the baselines of the observational sequence $(\Delta \mathrm{T})$.
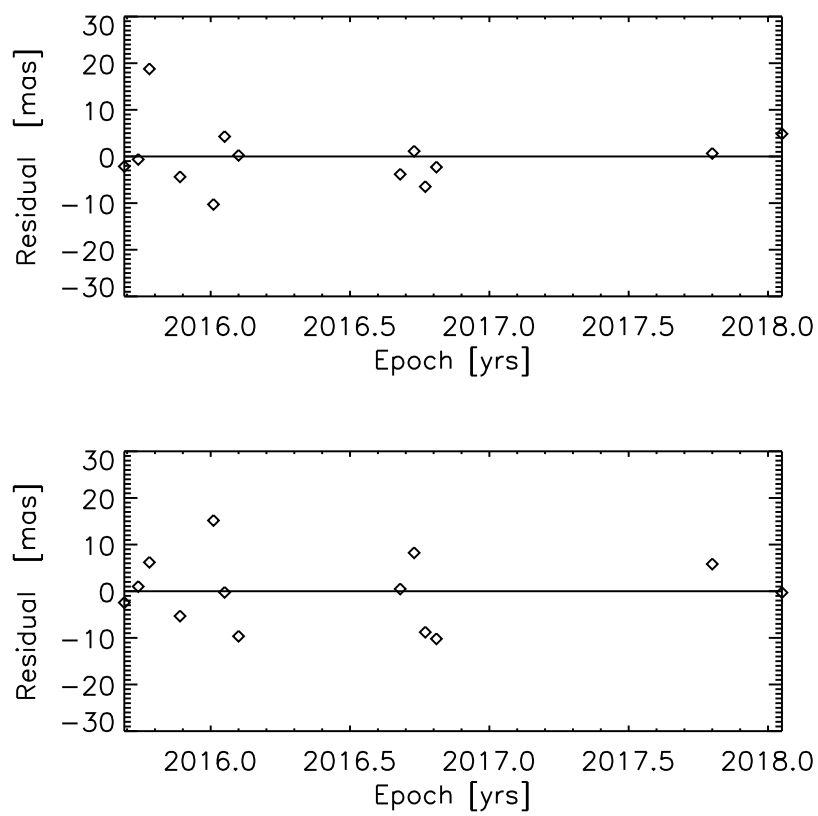

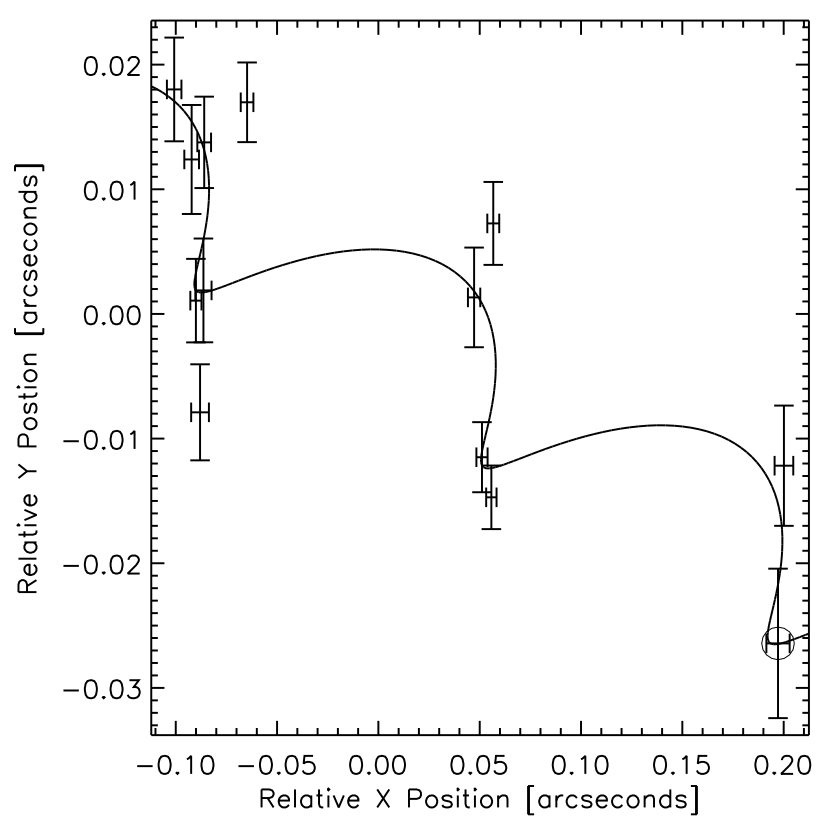

Fig. 3. Left panels: residuals in mas as a function of epoch for right ascension (bottom) and declination (top). Right panel: solution for the parallax determination. We show the example of CFHT-Hy-20, the other objects are plotted in Appendix A. The circled dot highlights the reference frame used for the determination of the parallax.

the difference of the median Gaia DR2 parallaxes of the common reference stars to the measured median parallax calculated from the observations. As can be seen from Table 2, this correction is always less than 1 mas and we added $20 \%$ of the correction to the formal parallax uncertainty in quadrature to obtain the quoted errors. The results are summarised in Tables 1-2 and in Fig. 3 we show the on-sky motion and the residuals for target CFHT-Hy20 along with the solution. The epochs of observations and plots of the solution and residuals for the other targets are included in Tables A.1-A.8 and Figs. A.1-A.4.

This is the first time that parallaxes have been determined with IO:I on the Liverpool robotic telescope and demonstrates that it is possible to derive useful ground-based parallaxes up to $\sim 50 \mathrm{pc}$. There remains an important sample of very cool nearby objects that are too faint for Gaia but possible on this telescopeand-instrument combination.

\section{Selection of Hyades member candidates}

In this section, we implemented the kinematic procedure described by Perryman et al. (1998), whose technique was orig- inally described in Jones (1971). This method determines the barycentre of the cluster and identifies potential members based on their velocities in space (Sect. 5.1).

We decided to use this method because it was specifically developed for the Hipparcos satellite, whose astrometry offers much higher accuracy than ground-based surveys. The advent of Gaia provides even more accurate parallaxes and proper motions for a significantly larger number of stars in the Galaxy.

\subsection{The kinematic method}

We applied the kinematic procedure of Perryman et al. (1998) to the sample of 126144 objects located within a radius of $70^{\circ}$ from the cluster centre at $(\mathrm{RA}, \mathrm{Dec})=(67,16)^{\circ}$. This method has been successfully applied to the Hyades using Hipparcos (Perryman et al. 1998; de Bruijne et al. 2001) and Gaia DR1 (Reino et al. 2018).

We implemented the Bayesian method using equations 17 and 18 of Luri et al. (2018) to transform Gaia parallaxes into distances. To determine the membership probability of all objects in the $70^{\circ}$ area, we calculated the space velocity of the Hyades 
Table 3. Positional and kinematics data of the Hyades cluster.

\begin{tabular}{|c|c|c|c|c|c|c|c|c|c|}
\hline \multirow[t]{2}{*}{ Selection } & \multirow[t]{2}{*}{$N$} & \multicolumn{3}{|c|}{$\boldsymbol{b}_{\mathrm{c}}(\mathrm{pc})$} & \multicolumn{3}{|c|}{$\boldsymbol{v}_{\mathrm{c}}\left(\mathrm{km} \mathrm{s}^{-1}\right)$} & \multirow{2}{*}{$\begin{array}{c}\text { Distance } \\
\text { pc }\end{array}$} & \multirow{2}{*}{$\begin{array}{l}\text { Velocity } \\
\mathrm{km} \mathrm{s}^{-1}\end{array}$} \\
\hline & & $b_{x}$ & $b_{y}$ & $b_{z}$ & $v_{x}$ & $v_{y}$ & $v_{z}$ & & \\
\hline & 154 & & 0 & & & 3 & 5 & 47.6 & 46 \\
\hline$r<10 \mathrm{pc}$ & 122 & $-43.83 \pm 0.18$ & $0.42 \pm 0.11$ & $-17.05 \pm 0.09$ & $-42.14 \pm 0.11$ & $-19.26 \pm 0.04$ & -1.12 & $47.03 \pm 0.20$ & $46.34 \pm 0.12$ \\
\hline$r<20 \mathrm{pc}$ & 168 & $-44.45 \pm 0.15$ & $0.26 \pm 0.10$ & $-17.16 \pm 0.08$ & $-42.19 \pm 0.10$ & $-19.25 \pm 0.03$ & $-1.14 \pm 0.05$ & $47.65 \pm 0.17$ & $46.39 \pm 0.11$ \\
\hline
\end{tabular}

Notes. First row shows the data obtained with the preliminary list of 154 Hyades members. Second (third) row display the results after recalculating cluster centre and velocity with objects from the final list closer than $10 \mathrm{pc}(20 \mathrm{pc})$ to the cluster centre.

Table 4. Summary of numbers of members in our work and from the various cross-matches with catalogues from previous studies (Sect. 5.2).

\begin{tabular}{lll}
\hline \hline Method & Members & Comments \\
\hline This paper & $85,381,568,710$ & within 3.1, 9, 18, 30 pc \\
Recovered in Perryman et al. (1998) & 129 out of 192 & Memb =1 from Perryman; 127 within 30 pc \\
Recovered in Perryman et al. (1998) & None & Memb= ? or 0 from Perryman \\
Recovered in Gaia Collaboration (2018a) & 503 out of 515 & all within 20 pc \\
Recovered in Reino et al. (2018) & 169 out of 187 & Gaia DR1; 159 within 30 pc \\
Recovered among pre-Gaia known members & $70,306,443,518$ & within 3.1, 9, 18, 30 pc from 749 \\
Recovered in BANYAN & $85,376,469,484$ & within 3.1, 9, 18, 30 pc (568 candidates using BANYAN) \\
\hline
\end{tabular}

cluster following Perryman et al. (1998). For the sake of clarity we sketch this method below. The Gaia mission provides high quality parallax measurements $(\pi)$, proper motions $\left(\mu_{\alpha} \cos \delta, \mu_{\delta}\right)$, and radial velocities $\left(V_{R}\right)$; the latter only for the brightest sources $(G \sim 5-13 \mathrm{mag})$. In a first step we calculate the cluster barycentre $\left(\boldsymbol{b}_{\mathrm{c}}\right)$ and space velocity $\left(\boldsymbol{v}_{\mathrm{c}}=v_{x}, v_{y}, v_{z}\right)$ using a preliminary set of Hyades members selected by Gaia Collaboration (2018a). We calculated these quantities using the standard expressions:

$\boldsymbol{b}_{\mathrm{c}}=\frac{\sum m_{i} \boldsymbol{b}_{i}}{\sum m_{i}}, \boldsymbol{v}_{\mathrm{c}}=\frac{\sum m_{i} \boldsymbol{v}_{i}}{\sum m_{i}}$,

where $\boldsymbol{b}_{i}=\left(d_{i} \cos \alpha_{i} \cos \delta_{i}, d_{i} \sin \alpha_{i} \cos \delta_{i}, d_{i} \sin \delta_{i}\right)$ is the positional vector for object $i$, with equatorial coordinates $\alpha_{i}$ and $\delta_{i}$ and located at a distance $d_{i}$ (measured in pc). The mass $m_{i}$ is estimated using predicted magnitudes and $G-J$ colours from the Padova and BT-Settl models (see Sect. 7.2 for more details). The mass is used in the calculation of the barycentre but as a weight. Assuming that binaries are distributed isotropically with respect to the centre then the barycentre should not change. We checked that this is the case assuming that all stars have a mass of $1 M_{\odot}$, which would largely account for binaries. This fact agrees with the conclusions of Perryman et al. (1998) and Reino et al. (2018) who concluded that "these results are rather insensitive to the weighting scheme adopted". In conclusion, binaries do impact on the total mass and the mass function (Sect. 7.2) but not the barycentre. The velocity vectors $\left(\boldsymbol{v}_{i}\right)$ are calculated using the object transverse and radial velocities with the equation:

$\left(\begin{array}{c}v_{i x} \\ v_{i y} \\ v_{i z}\end{array}\right)=\mathbf{R}_{i}\left(\begin{array}{c}V_{i \alpha *} \\ V_{i \delta} \\ V_{i R}\end{array}\right)$,

where $V_{i \alpha *}=\mu_{i \alpha *} A_{v} / \pi_{i}, V_{i \delta}=\mu_{i \delta} A_{v} / \pi_{i}$, and $V_{i R}$ are the observed transverse and radial velocities, with $A_{v}=4.74047 \mathrm{~km} \mathrm{yr} \mathrm{s}^{-1}$. The matrix $\mathbf{R}_{i}$ is given by:

$\mathbf{R}_{i}=\left(\begin{array}{ccc}-\sin \alpha_{i} & -\sin \delta_{i} \cos \alpha_{i} & \cos \delta_{i} \cos \alpha_{i} \\ \cos \alpha & -\sin \delta_{i} \sin \alpha_{i} & \cos \delta_{i} \sin \alpha_{i} \\ 0 & \cos \delta_{i} & \sin \delta_{i}\end{array}\right)$,
In a second step, we use $\boldsymbol{v}_{\mathrm{c}}$ to select objects in the Gaia database with motions consistent with the cluster. To carry out this task, we estimate the expected transverse and radial velocities at the position of each object:

$$
\left(\begin{array}{c}
V_{i \alpha *}^{e} \\
V_{i \delta}^{e} \\
V_{i R}^{e}
\end{array}\right)=\mathbf{R}_{i}^{-1}\left(\begin{array}{c}
v_{x} \\
v_{y} \\
v_{z}
\end{array}\right)
$$

where $\mathbf{R}_{i}^{-1}$ is actually the transpose matrix of $\mathbf{R}_{i}$ from Eq. (3). We define the vector $z_{i}$ as the difference vector between the observed and expected transverse and radial velocities. We need to calculate two covariance matrices, one associated with the observed transverse or radial velocities and the other associated with the expected ones (see detailed explanation in Perryman et al. 1998). Assuming that these velocities are statistically independent, the sum of their two covariance matrices $\Sigma$ describes the combined confidence region and the parameter:

$c=z^{T} \Sigma^{-1} z$

is a $\chi^{2}$ statistical test with three degrees of freedom (DOF). As not all the objects in Gaia have radial velocities we adapted the method to work also with transverse velocities only. For sources without radial velocity the test has only two d.o.f. We selected as good candidates those objects with velocities within $4.4171 \sigma$ of common cluster motion, which correspond to a $\mathrm{p}$ value of 0.99999. Thus for three d.o.f. (objects with radial velocity measured), all sources with $c<25.9$ are considered as Hyades members, while for objects without radial velocity in Gaia data, that is two DOF statistic, the threshold is 23.03.

From the preliminary list of Hyades members from Gaia Collaboration (2018a) we calculated $\boldsymbol{b}_{\mathrm{c}}$ and $\boldsymbol{v}_{\mathrm{c}}$, as explained above, and checked whether all sources in that list can be considered as good members of the cluster using the value of the $c$ parameter (Eq. (5)). Discarding those with values of $c$ larger than the chosen threshold and then recalculating new values for $\boldsymbol{b}_{\mathrm{c}}$ and $\boldsymbol{v}_{\mathrm{c}}$. This procedure is repeated until no further objects are discarded. At the end of this process we have 154 objects and the final values of $\boldsymbol{v}_{\mathrm{c}}$ are employed to assess which objects from 

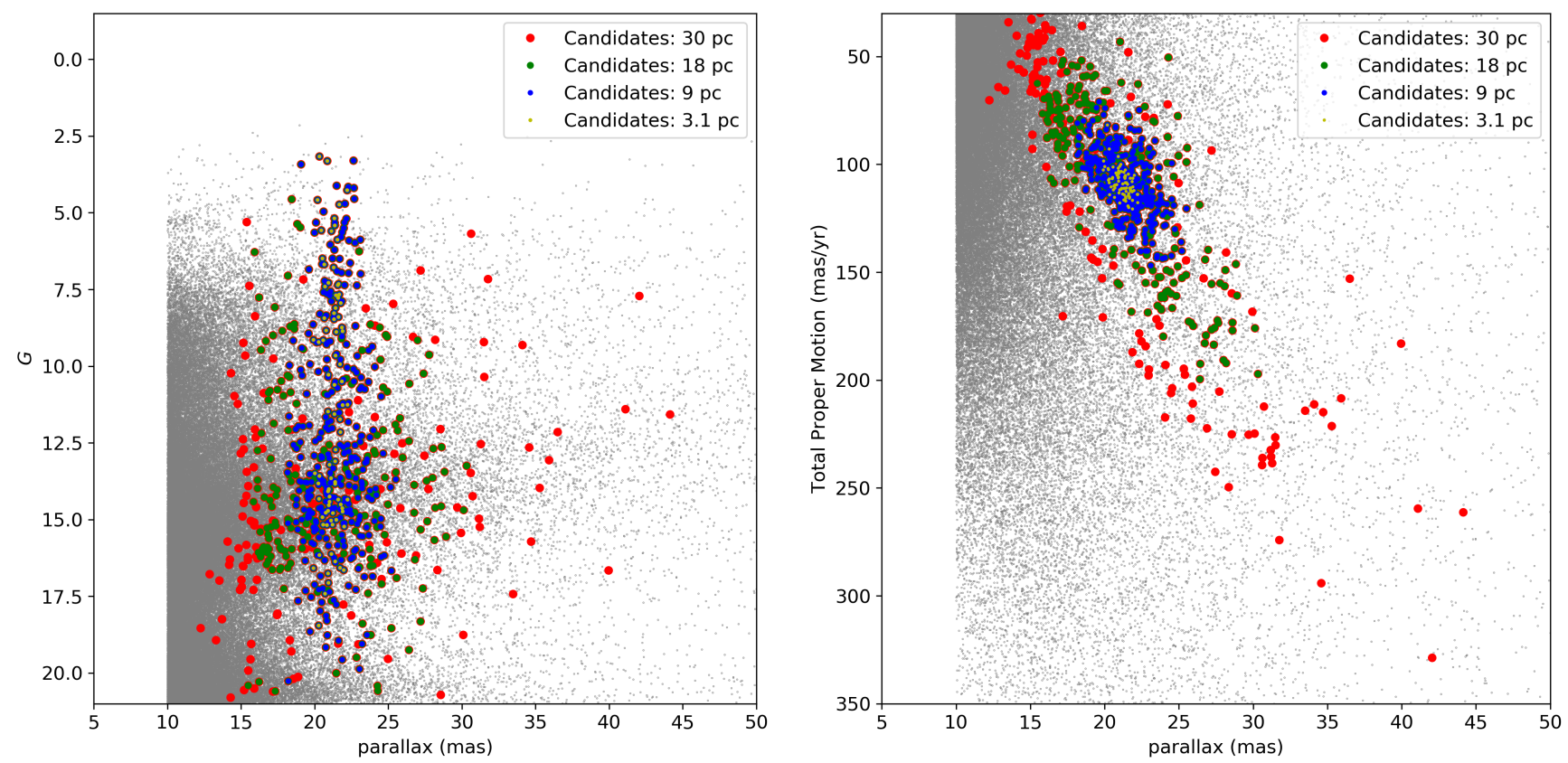

Fig. 4. Left panel: parallax as a function of the $G$-band magnitudes from Gaia DR2 for candidates within radii of $3.1 \mathrm{pc}$ (yellow), $9 \mathrm{pc}$ (blue), $18 \mathrm{pc}$ (green), and $30 \mathrm{pc}$ (red) from the cluster centre. Right panel: parallax as a function of the total proper motion from Gaia DR2. We added as small grey dots the full Gaia catalogue over the $70^{\circ}$ radius.

Gaia catalogue can be considered as bona-fide Hyades members. In Table 3 we list the barycentre and cluster velocity in Galactic coordinates. We applied this procedure to the list of 126 motions to the cluster. This final list is then used to re-estimate $\boldsymbol{b}_{\mathrm{c}}$ and $\boldsymbol{v}_{\mathrm{c}}$ (Table 3 ). The cluster centre velocity obtained when we use objects located in a radius of $10 \mathrm{pc}$ from the cluster centre is employed to carry out a second iteration with the final list of 1764 objects checking that all of them continue to be considered as good Hyades members.

As discussed at length in Röser et al. (2011), most of the members of the cluster lie within its tidal radius. In the case of the Hyades, all objects within $9 \mathrm{pc}$ are most likely bound while the candidates up to $18 \mathrm{pc}$ most likely belong to its halo. Kinematic candidates in the $18-30 \mathrm{pc}$ might belong to the Hyades moving group (Boss 1908; Eggen 1958; Zuckerman \& Song 2004; Famaey et al. 2007) but we expect a significant level of contamination. We do not consider the candidates beyond the $30 \mathrm{pc}$ limit in this work (710 sources). We limit our analysis to the core $(3.1 \mathrm{pc})$, tidal radius $(9 \mathrm{pc})$, halo $(18 \mathrm{pc})$, and up to $30 \mathrm{pc}$ from the cluster centre in 3D space to allow for direct comparison with the work of Röser et al. (2011). The kinematic procedure returned $85,381,568$, and 710 sources within $3.1,9$, 18 , and $30 \mathrm{pc}$, respectively (Figs. 4-5). The large area at around $\mathrm{RA}=90-100^{\circ}$ and declinations around zero containing candidates further than $30 \mathrm{pc}$ from the centre of the Hyades corresponds to clusters in the galactic plane, and possibly to Platais 6 , whose extension on the sky is estimated to be 250 arcmin (Dias et al. 2002, 2006, 2014, 2018).

We display several colour-magnitude diagrams in Appendix B, showing combinations of Gaia magnitudes with other large-scale surveys (Figs. B.1-B.3). We display all candidates identified in this work as black dots and highlight the members located within $3.1,9,18$, and $30 \mathrm{pc}$ with yellow, blue, green, and red symbols, respectively. The sequences drawn in those colour-magnitude diagram represent benchmark sequences at an age of $650 \mathrm{Myr}$, which are key to compare with other clusters and members of moving groups.

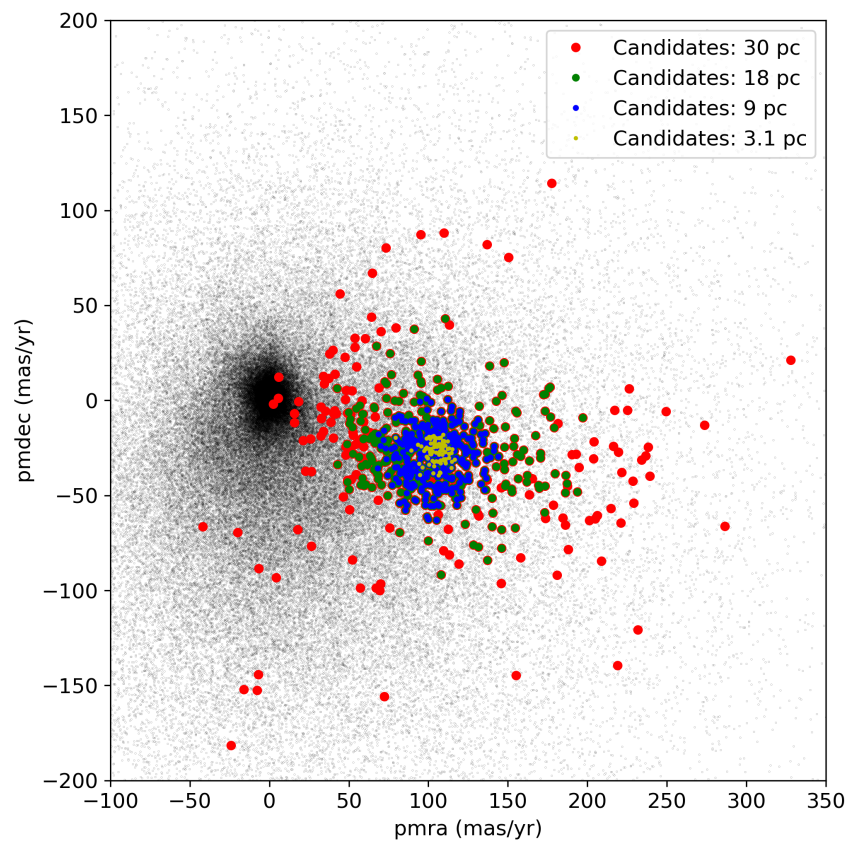

Fig. 5. Vector point diagram with proper motions in right ascension and declination for all sources in the full $70^{\circ}$ catalogue (grey). We overplot kinematic candidate members belonging to the Hyades and located within radii of $3.1 \mathrm{pc}$ (yellow), $9 \mathrm{pc}$ (blue), $18 \mathrm{pc}$ (green), and $30 \mathrm{pc}$ (red).

\subsection{Comparison with previous Hyades surveys}

\subsubsection{Hipparcos catalogue from Perryman et al. (1998)}

We cross-correlated our sample of Hyades candidate members with the Hipparcos catalogue (Perryman et al. 1998), which is divided up into three groups: 192 members $(\mathrm{Memb}=1), 20$ probable members $(\mathrm{Memb}=$ ?), and 60 non-members $(\mathrm{Memb}=0)$. The Gaia DR2 catalogue contains 183 of the 192 members (>95\% completeness) but only 13 of the 69 probable members 
$(18.8 \%)$ and 31 of the 60 non-members $(51.7 \%)$. We recovered 129 sources of the 192 objects classified as members $(\mathrm{Memb}=1$ in their table), see Table 4 in our full list, including 127 being within $30 \mathrm{pc}$. The remaining 63 sources do not satisfy the criteria of the kinematic method because they have "c" indices larger than 25.9 or 23.03 depending on the availability of Gaia radial velocity. None of the other candidates reported by Perryman et al. (1998) as probable members or likely nonmembers in their table have counterparts in our catalogue of candidates within $70^{\circ}$ from the cluster centre.

We expect some difference because the cluster parameters derived from Gaia DR2 are slightly different from those inferred by HiPPARcos: from our $10 \mathrm{pc}$ sample, we find that a mean distance and velocity of the cluster is $47.03 \pm 0.20 \mathrm{pc}$ and $46.38 \pm 0.12 \mathrm{~km} \mathrm{~s}^{-1}$, respectively, compared to $46.34 \pm 0.27 \mathrm{pc}$ and $45.93 \pm 0.23 \mathrm{~km} \mathrm{~s}^{-1}$ for the Hipparcos $10 \mathrm{pc}$ sample (Table 3 of Perryman et al. 1998). We note that the global Gaia zero point is -0.03 mas but it varies upon position in the sky by 0.15 to -0.15 mas (Lindegren et al. 2018). This correction is negligible compared to the average parallax of the Hyades ( 21-22 mas). The Gaia-Hipparcos offset is -0.118 mas (Arenou et al. 2018), which means that the distance of the Hyades from Hipparcos put on the Gaia system would equate into 46.595 pc, close to our estimate using the $10 \mathrm{pc}$ sample. This is the distance we adopt in this work. The distance from Gaia Collaboration (2018a) makes use of all kinematic members up to 16 pc (Sect. 4 in Gaia Collaboration 2017) and should therefore be closer to our 20 pc estimate, which is indeed the case within $1 \sigma$. This difference will lead to some variation in the numbers of kinematic members.

\subsubsection{Gaia DR2 catalogue (Gaia Collaboration 2018a)}

We cross-correlated our sample with the list of 515 member candidates from Gaia Collaboration (2018a). The matching was based on the SourceID parameter to avoid any mis-matches due to positional matching problems. We recovered 503 out of 515 objects that lie in a radius of approximately $16 \mathrm{pc}$, the limit set intrinsically by the procedure described in Gaia Collaboration (2018a). We find that only a small number of sources of Gaia Collaboration (2018a) are not in our sample of member candidates. The small difference in numbers is most likely due to the divergence between our space velocity and distance estimates. Furthermore, Gaia Collaboration (2018a) clearly stated in their Sect. 2 that they "selected the most precise data, without trying to reach completeness". This is particularly the case in the low-mass and sub-stellar regimes where Gaia gives larger astrometric errors but remains nonetheless reliable. We noticed this effect when comparing their candidates with our sample in the various colour-magnitude diagrams presented in this work.

\subsubsection{TGAS catalogue from Reino et al. (2018)}

We collected the Gaia DR2 SourceID of the 251 candidates identified by Reino et al. (2018) based on Tycho-Gaia DR1 catalogue. Reino et al. (2018) kept 187 out of 251 candidates as bona-fide members while the other were rejected on the basis of their modelled parallaxes and standard errors. We found that 242 of the 251 have Gaia DR2 counterparts. We checked that the nine missing objects have entries in the second release of Gaia without parallax and proper motion. We recovered 169 as potential members of the Hyades, including 159 within $30 \mathrm{pc}$ from the centre (Table 4).

\subsubsection{Known members}

We cross-correlated our sample with the list of 749 known members with Gaia DR2 parallaxes and located within $70^{\circ}$ of the cluster. We conducted the cross-correlation with the SourceID keyword as before. In the full $70^{\circ}$, we retrieved 527 pre-Gaia known members. We recover eight of the ten brightest Hyades members, except $\Theta^{1}$ Tauri (G5III; c = 398.325; Keenan \& McNeil 1989) and 71 Tauri (F0V; c = 85.39), whose Gaia parallaxes and proper motions confirm their membership. The former is a known spectroscopic binary and the latter is a known variable star. These eight bright members are the brightest sources in the colour-magnitude diagrams showing Gaia photometry (e.g. Fig. 6). All these sources lie within $6 \mathrm{pc}$ from the cluster centre. Therefore, $749-527=222$ previouslyknown members are rejected by the kinematic method because their "c" indices are larger than the maximum values allowed for membership even though their proper motions, parallaxes and magnitudes maybe consistent with other Hyades members. We conclude that the level of contamination from earlier surveys using the convergent point method is about $30 \%$, consistent with ground-based photometric surveys in the Pleiades (Bouvier et al. 1998; Moraux et al. 2001) and Alpha Persei (Barrado y Navascués et al. 2002; Lodieu et al. 2005). We recovered 140, 312, 446, and 522 pre-Gaia known members within $3.1,9,18,30 \mathrm{pc}$ from the cluster centre, respectively (Table 4). Hence, the level of completeness of previous surveys is decreasing with larger radii, from $80.9 \%$ in the $3.1 \mathrm{pc}$ radius to $73.6 \%$ in the halo.

\subsection{Comparison with BANYAN}

Malo et al. (2013) developed a new method based on a Bayesian analysis to identify new members of nearby young kinematic groups and assess their membership probability. This method, the Bayesian Analysis for Nearby Young AssociatioNs (BANYAN), was later updated by Gagné et al. (2014) and further improved by Gagné et al. (2018a) to model the 6D space with multi-variate Gaussians to take advantage of Gaia. This algorithm has been successfully employed to revise the membership of known members of young moving groups and also identify new candidates in a series of papers (Artigau et al. 2015; Boucher et al. 2016; Gagné et al. 2015b,a, 2017, 2018b; Malo et al. 2014a,b).

The new BANYAN $\Sigma$ algorithm is freely available ${ }^{2}$ and includes the Hyades in the list of nearby associations. We used the IDL version which allowed us to calculate probabilities using proper motions, parallaxes and radial velocities or sub-sets of those parameters. We did not incorporate any extra constraints, for example indicators of youth. We ran this algorithm on the full $70^{\circ}$ catalogue (126144 objects), and 568 objects were returned with a most probable Bayesian hypothesis of being a Hyades member. The cluster parameters given in Table 9 of Gagné et al. (2018a) are the updated parameters of the Hyades combining Gaia DR1 (Gaia Collaboration 2017) with member candidates from Eggen (1969), Perryman et al. (1998), Mermilliod et al. (2009), and Röser et al. (2011).

We cross-correlated our full sample of 1764 sources with the 568 candidates from BANYAN and found 484 objects in common. Limiting the analysis to the radii of $3.1,9,18$, and $30 \mathrm{pc}$ from the cluster centre, we have $85,376,469$, and 484 in common with BANYAN, respectively. The remaining 568-484 = 84

\footnotetext{
2 wWw. exoplanetes.umontreal.ca/banyan/banyansigma.php
} 
Table 5. Hyades WDs in Gaia DR2: single WDs are at the top and binary WDs at the bottom.

\begin{tabular}{|c|c|c|c|c|c|c|c|c|c|}
\hline SourceID & Name & $\begin{array}{c}\text { RA } \\
\text { (deg) }\end{array}$ & $\begin{array}{c}\text { Dec } \\
(\mathrm{deg})\end{array}$ & $\begin{array}{c}\text { Plx } \\
\text { (mas) }\end{array}$ & $\begin{array}{c}\text { pmRA } \\
\left(\mathrm{mas} \mathrm{yr}^{-1}\right)\end{array}$ & $\begin{array}{c}\mathrm{pmDEC} \\
\left(\mathrm{mas} \mathrm{yr}^{-1}\right)\end{array}$ & $\begin{array}{c}B p \\
(\mathrm{mag})\end{array}$ & $\begin{array}{c}R p \\
(\mathrm{mag})\end{array}$ & $\begin{array}{c}G \\
(\mathrm{mag})\end{array}$ \\
\hline 45980377978968064 & EGGR29 & 62.3709338 & 17.1316806 & $19.9402 \pm 0.0931$ & $111.4542 \pm 0.2682$ & $-22.0215 \pm 0.1464$ & 15.302 & 15.460 & 15.378 \\
\hline 3294248609046258048 & HZ14 & 70.2576501 & 10.9943984 & $20.2471 \pm 0.0510$ & $91.3544 \pm 0.0937$ & $-10.6426 \pm 0.0497$ & 13.669 & 14.108 & 13.864 \\
\hline 3306722607119077120 & $\mathrm{HZ7}$ & 68.4377818 & 12.7111484 & $21.1400 \pm 0.0616$ & $99.0219 \pm 0.1237$ & $-14.3138 \pm 0.0711$ & 14.122 & 14.437 & 14.262 \\
\hline 3308403897837092992 & EGGR316 & 70.0999149 & 13.9793215 & $21.7321 \pm 0.0563$ & $95.2761 \pm 0.1097$ & $-20.6871 \pm 0.0512$ & 14.810 & 15.006 & 14.932 \\
\hline 218783542413339648 & GD52 & 58.0005259 & 34.1241725 & $23.5584 \pm 0.0457$ & $145.9926 \pm 0.0980$ & $-77.6952 \pm 0.0668$ & 15.153 & 15.257 & 15.202 \\
\hline 3313714023603261568 & EEGR37 & 67.1646712 & 16.9699097 & $20.8952 \pm 0.0567$ & $102.6919 \pm 0.1147$ & $-26.8853 \pm 0.0681$ & 13.913 & 14.288 & 14.075 \\
\hline 3313606340183243136 & EGGR36 & 65.9825865 & 16.3540809 & $22.2272 \pm 0.0519$ & $114.4115 \pm 0.1034$ & $-27.7153 \pm 0.0792$ & 14.219 & 14.505 & 14.347 \\
\hline 39305036729495936 & HG7-85 & 60.9260025 & 14.9912221 & $24.0527 \pm 0.0541$ & $141.1900 \pm 0.1073$ & $-24.0698 \pm 0.0843$ & 14.984 & 15.112 & 15.039 \\
\hline 3302846072717868416 & HZ4 & 58.8423743 & 9.7883468 & $28.5890 \pm 0.0536$ & $173.2722 \pm 0.1072$ & $-5.5099 \pm 0.0788$ & 14.511 & 14.644 & 14.564 \\
\hline 43789772861265792 & V471-Tau & 57.6046015 & 17.2464125 & $20.9569 \pm 0.0440$ & $127.4578 \pm 0.0956$ & $-22.4774 \pm 0.0643$ & 9.678 & 8.591 & 9.200 \\
\hline 3310615565476268032 & HD27483 & 65.2201424 & 13.8643726 & $21.0518 \pm 0.0769$ & $106.9751 \pm 0.1947$ & $-12.7011 \pm 0.1071$ & 6.284 & 5.683 & 6.033 \\
\hline 3314232855652895104 & HZ9 & 68.0994836 & 17.7505941 & $23.2618 \pm 0.0373$ & $109.8651 \pm 0.0661$ & $-34.5059 \pm 0.0419$ & 14.030 & 12.445 & 13.362 \\
\hline 3311810043124387712 & LP474-185 & 63.4688348 & 15.3649605 & $23.6172 \pm 0.5733$ & $116.2583 \pm 1.0037$ & $-24.2140 \pm 0.7910$ & 15.417 & 12.495 & 14.257 \\
\hline
\end{tabular}

Table 6. Derived parameters for the eight single DA WD members in the Hyades, considered for the age determination.

\begin{tabular}{|c|c|c|c|c|c|c|c|c|}
\hline SourceID & $\begin{array}{l}M_{\mathrm{WD}} \\
\left(M_{\odot}\right) \\
\end{array}$ & $\begin{array}{l}M_{\mathrm{MS}} \\
\left(M_{\odot}\right)\end{array}$ & $\begin{array}{c}\text { Time }_{W D} \\
(\mathrm{Myr})\end{array}$ & $\begin{array}{c}\text { Time }_{\mathrm{MS}} \\
(\mathrm{Myr})\end{array}$ & $\begin{array}{c}\text { Time }_{\text {total }} \\
\text { (Myr) }\end{array}$ & SpT & $\begin{array}{l}T_{\text {eff }} \\
(\mathrm{K})\end{array}$ & $\begin{array}{l}\log g \\
(\operatorname{dex})\end{array}$ \\
\hline 45980377978968064 & 0.826 & $3.823_{-0.478}^{+0.637}$ & 355.0 & $236.8_{-80.2}^{+107.2}$ & $591.8_{-80.2}^{+107.2}$ & DA3.2 & $15810 \pm 288$ & $8.38 \pm 0.05$ \\
\hline 3294248609046258048 & 0.708 & $2.9657_{-0.343}^{+0.412}$ & 20.4 & $487.8_{-152.7}^{+214.6}$ & $508.2_{-152.7}^{+214.6}$ & DA1.8 & $27540 \pm 403$ & $8.15 \pm 0.05$ \\
\hline 3306722607119077120 & 0.666 & $2.673_{-0.299}^{+0.349}$ & 78.8 & $664.7_{-203.9}^{+272.6}$ & $743.4_{-203.9}^{+27.6}$ & DA2.3 & $21890 \pm 346$ & $8.11 \pm 0.05$ \\
\hline 218783542413339648 & 0.838 & $4.003_{-0.508}^{+0.692}$ & 475.2 & $208.5_{-71.3}^{+95.2}$ & $683.7_{-71.3}^{+95.2}$ & DA3.4 & $14820 \pm 350$ & $8.31 \pm 0.05$ \\
\hline 3313714023603261568 & 0.691 & $2.863_{-0.327}^{+0.388}$ & 44.8 & $542.8_{-169.8}^{+232.9}$ & $587.6_{-169.8}^{+23.9}$ & DA2.0 & $25130 \pm 381$ & $8.12 \pm 0.05$ \\
\hline 3313606340183243136 & 0.693 & $2.879_{-0.330}^{+0.391}$ & 112.8 & $533.7_{-167.0}^{+229.8}$ & $646.6_{-167.0}^{+229.8}$ & DA2.5 & $20010 \pm 315$ & $8.13 \pm 0.05$ \\
\hline 39305036729495936 & 0.816 & $3.693_{-0.456}^{+0.598}$ & 400.4 & $260.2_{-87.0}^{+117.1}$ & $660.6_{-87.0}^{+117.1}$ & DA & $15131 \pm 209$ & $8.48 \pm 0.02$ \\
\hline 3302846072717868416 & 0.780 & $3.373_{-0.405}^{+0.510}$ & 359.2 & $336.1_{-109.3}^{+150.0}$ & $695.2_{-109.3}^{+150.0}$ & DA 3.4 & $14670 \pm 377$ & $8.30 \pm 0.05$ \\
\hline
\end{tabular}

objects from BANYAN are not in our list. About a quarter of these sources have right ascension below $60^{\circ}$, lying in the tail of members identified by BANYAN (Table 4). The differing candidate lists are most likely the result of the very different membership allocation procedures and also the different distance and space velocities between HiPPARcos/BANYAN (Perryman et al. 1998; Gagné et al. 2018a) and this work.

\section{White dwarfs}

One striking feature in the Hertzsprung-Russell diagrams displayed in Fig. 17 of Gaia Collaboration (2018a) is the presence of white dwarf (WD) members at around $B p-R p<0$ and $G \sim 14-16 \mathrm{mag}$. The existence of WDs in the Hyades has been known since the discovery of ten WDs by von Hippel (1998). Seven of these "classical" WD are single while three are binaries (Table 5) and one of them a doubtful member due to its distance and tangential velocity (Weidemann et al. 1992). Schilbach \& Röser (2012) presented a compendium of WD candidates in the Hyades, including the ten classical WDs (von Hippel 1998) and three new sub-groups: one with 12 new potential Hyades WDs (running number from 11 to 22) and two additional ones with most likely non-members (running number from 23 to 37 ).

We cross-matched this list of 37 WD candidates from Schilbach \& Röser (2012) with our Gaia DR2 and recovered 13 WDs previously published in the literature (Table 5). All the ten classical WDs (seven singles and three binaries) from von Hippel (1998) are confirmed as members based on Gaia parallaxes and proper motions and common to the sequence of
WDs from Gaia Collaboration (2018a). We note that the three classical WD binaries are unresolved in Gaia because they have orbital periods less than about 3 days. Three other WDs from the Gaia sample are common to the sub-group of new WD members in Table 1 of Schilbach \& Röser (2012). The remaining 37-15 = 22 candidates in Schilbach \& Röser (2012) are rejected as members based on their parallaxes and proper motions. All the three brightest WDs are binaries and appear much redder in the Gaia colours (bottom panels in Fig. 6). The WD+dM binary (EGGR 38; HZ9) lies between the WD sequence and the cluster main sequence in the $(B p-R p, R p)$ colour-magnitude diagram. The other two classical binaries lie on the cluster main-sequence: one is a known WD+F6 binary (HR 1358; HD 27483) and the other one is the known eclipsing binary of Algol type (WD+K2) V 471 Tau (Fig. 6). We find one more binary, LP 474-185 Schilbach \& Röser (2012), based on Gaia photometry.

Table 5 lists all the binary and single WDs belonging to the Hyades. Of the nine single stars in Table 5, we considered only eight with a pure hydrogen atmosphere (DA spectral class) to determine the cluster age. The other one, EGGR 316, was excluded because it is a DBA with mixed $\mathrm{H} / \mathrm{He}$ atmosphere (Bergeron et al. 2011). In Fig. 7, we compare the position of the eight single DA WDs in the $\left(G_{\mathrm{BP}}-G_{\mathrm{RP}}, M_{G}\right)$ colour-absolute magnitude diagram with the cooling tracks of DA white dwarfs with H-thick envelopes $\left(M_{\mathrm{H}} / M_{\star}=10^{-4}\right)$ from Bergeron et al. $(2011)^{3}$. From cubic spline interpolation of the models, we

\footnotetext{
3 http://www .astro.umontreal.ca/ bergeron/

CoolingModels/, see also Tremblay et al. (2012) and references therein.
} 

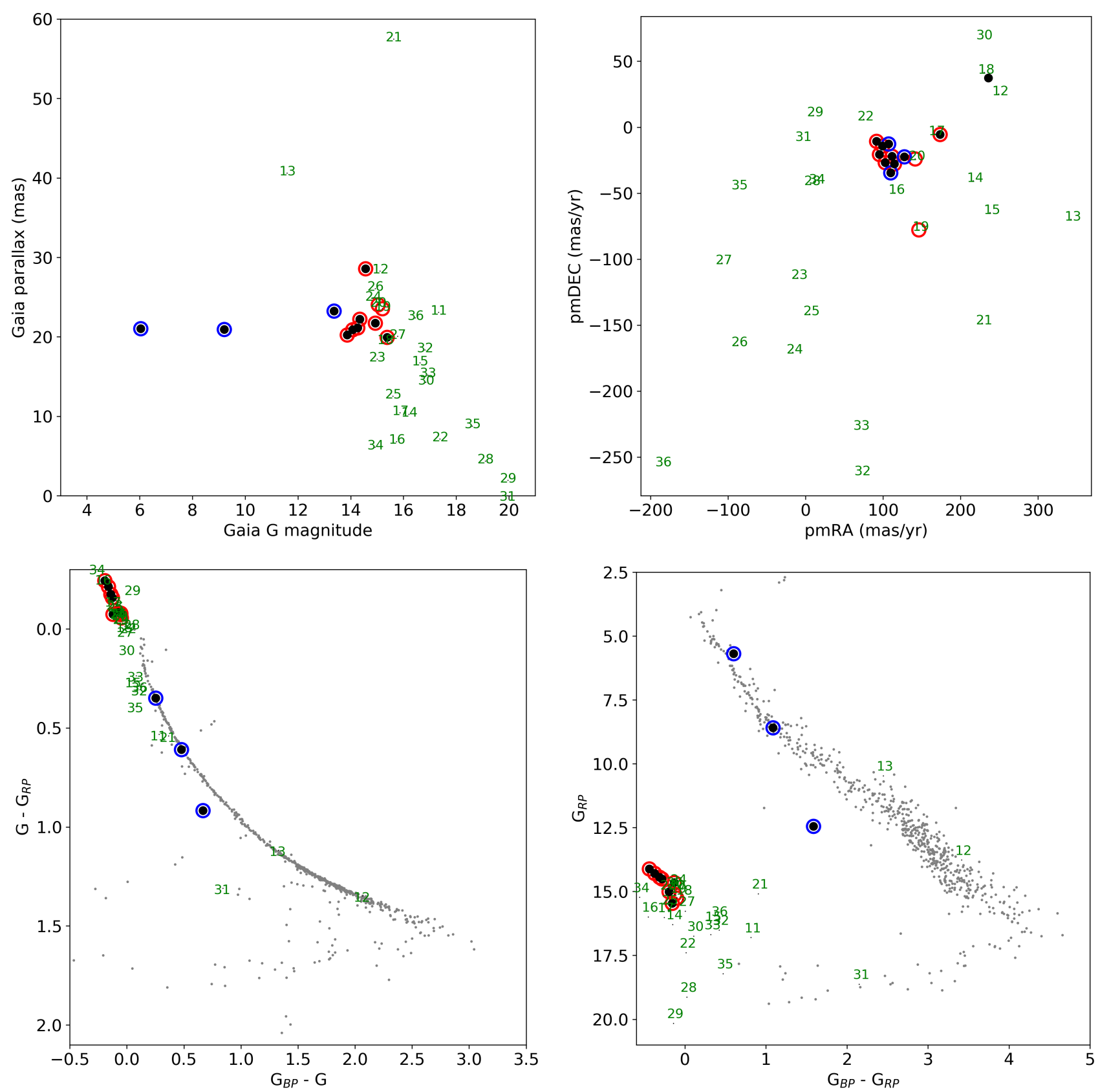

Fig. 6. Top left: parallaxes of Hyades WD candidates as a function of Gaia magnitude. Top right: vector point diagrams for Hyades WD candidates. Bottom left: $\left(G_{\mathrm{BP}}-G, G-G_{\mathrm{RP}}\right)$ colour-colour magnitude of all candidates in our $30 \mathrm{pc}$ sample. Bottom right: $\left(G_{\mathrm{BP}}-G_{\mathrm{RP}}, G_{\mathrm{RP}}\right)$ colour-magnitude magnitude for our $30 \mathrm{pc}$ sample. The classical WDs from von Hippel (1998) are shown as black dots. The candidates from Schilbach \& Röser (2012) are highlighted with their ID number. The Hyades single and binary WDs from Gaia Collaboration (2018a) are depicted as red and blue circles, respectively.

determined the basic physical parameters of each WD: effective temperature, surface gravity, mass and cooling age. After having verified that the effective temperatures and surface gravities $(\log g)$ agree with the known spectroscopic values from the literature, we used the initial-to-final mass relation (IFMR) of El-Badry et al. (2018) to derive the mass of each WD progenitor. Then, for each WD progenitor, we used the Padova evolutionary models of massive stars (Bressan et al. 2012) ${ }^{4}$ with nearly solar abundances $(Z=0.017, Y=0.279)$ to compute the time needed to evolve from the pre-main-sequence to the first thermal pulse in the asymptotic giant branch.

\footnotetext{
4 http://people.sissa.it/ sbressan/CAF09_V1.2S_M36_ LT/, see also Tang et al. (2014)
}

The results of our computations are summarised in Table 6 . The largest source of error comes from the IFMR: the uncertainty on the initial mass implies an uncertainty on the mainsequence evolutionary time. Compared to this uncertainty, the Gaia photometric and parallax errors are negligible for these relatively bright stars. When considering all eight single WDs in Table 6, we infer an age of $640_{-49}^{+67} \mathrm{Myr}$ for the Hyades cluster.

This age agrees with the early estimate from the cooling age of the WD by De Gennaro et al. (2009), who quoted $648 \pm 45$ Myr. Those values are also consistent with the mean age of the cluster derived from isochrone fitting with convective overshoot $(625 \pm 50 \mathrm{Myr}$; Maeder \& Mermilliod 1981; Mermilliod 1981) and the lithium depletion boundary method (650 \pm 70 Myr; Lodieu et al. 2018; Martín et al. 2018). However, 


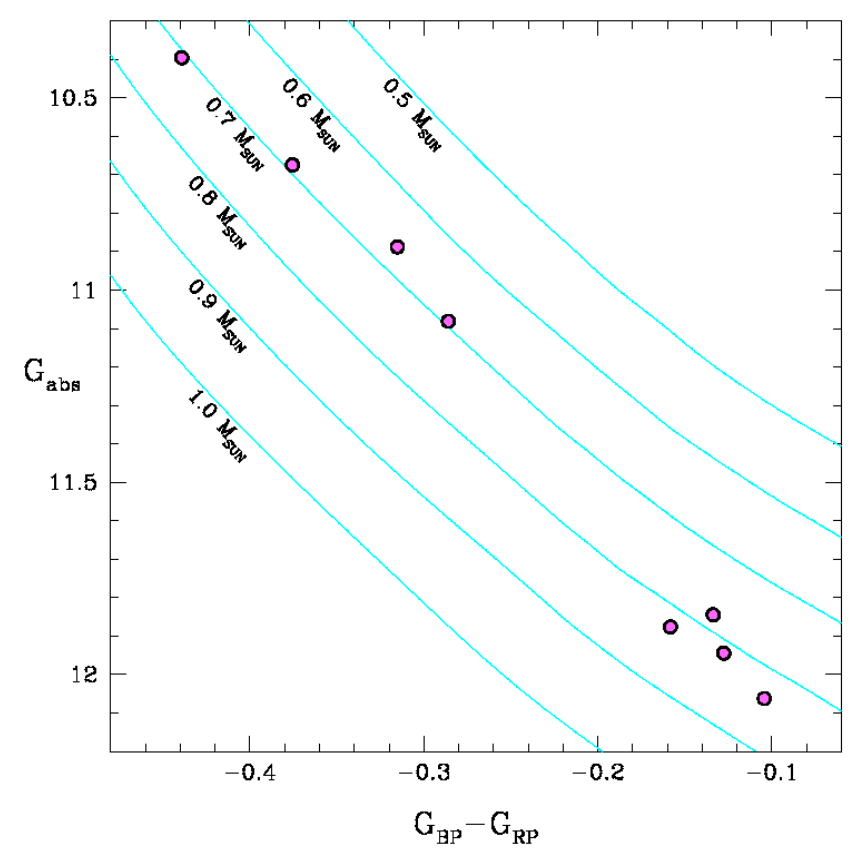

Fig. 7. Absolute magnitude of the Hyades white dwarfs as a function of the $G_{\mathrm{BP}}-G_{\mathrm{RP}}$ colour. The error bars, smaller than the symbols, are always lower than 0.011 and 0.008 in absolute magnitude and $G_{\mathrm{BP}}-G_{\mathrm{RP}}$ colour respectively. The WD cooling tracks of Bergeron et al. (2011) for various masses are shown in light blue (see text for more details).

it is on the lower side of older ages determined from evolutionary models with enhanced convective overshooting (upper limit of $1.2 \mathrm{Gyr}$; Mazzei \& Pigatto 1988) and models with rotation (750 \pm 100 Myr; Brandt \& Huang 2015).

After the first submission of this paper, a new article by Salaris \& Bedin (2018) makes use of the same sample of single DA WDs. Using a recent estimate of the cluster age from the main-sequence turn off, these authors derived the WD progenitor masses and compared the Hyades IFMR with the global IFMR. It is basically the opposite with respect to what we have done.

\section{The luminosity and mass functions}

In this section, we present the luminosity function as a function of radius from the cluster centre and derive the associated mass function including all stellar and sub-stellar members.

\subsection{Luminosity function}

We derive the system luminosity function from our sample of Hyades candidates identified using Gaia DR2 data. This sample contains a total of $85,381,568$, and 710 objects in $3.1,9,18$, and $30 \mathrm{pc}$ from the cluster centre, respectively. We did not attempt to correct the system luminosity function for binaries and postpone this analysis to later Gaia releases where astrometric parameters of multiple systems will be incorporated. The impact of binaries is not expected to be negligible as $20-40 \%$ of candidates are predicted to be in multiple systems (Duchêne et al. 2013; Gunn et al. 1988; Reid \& Mahoney 2000; Reino et al. 2018; Röser et al. 2011). The multiplicity varies as a function of mass, with high-mass stars being more likely in multiple systems. However, the precise impact on the luminosity function is difficult to estimate because multiple systems should be investigated over a wide range of separation and mass ratios.
In Fig. 8, we display the system luminosity function, that is the number of objects per absolute magnitude bins $\left(M_{G}\right)$, with bin width of one mag scaled to a volume of one cubic parsec for four different annuli from the cluster centre. We choose to display the regions within $3.1 \mathrm{pc}$ (core radius), 3.1-9 pc (tidal radius), 9-18 pc (halo), and $18-30 \mathrm{pc}$. We do not apply any correction to the luminosity function or plot the error bars. However, we note that two of the ten brightest known pre-Gaia members are not recovered in our $30 \mathrm{pc}$ sample for the reasons discussed in Sect. 5.2.4. The LF is also affected to some level by incompleteness at the faint end of Gaia because a member at $10 \mathrm{pc}$ will be about one magnitude fainter than a member at the centre of the cluster. However, the Gaia sample is mainly complete down to the hydrogen-burning limit with an uncertainty of $\pm 0.01 M_{\odot}$ because we showed that all brown dwarfs with lithium are not catalogued in Gaia DR2 (Tables 1-2). Models predict that a $0.072 M_{\odot}$ Hyades member has $G \sim 19.65$ mag, making it detectable up to the tidal radius. Further discussion on the incompleteness is provided in the next section (Sect. 7.2). However, the completeness of Gaia DR2 is a function of magnitude, position on the sky density (Sect. 3 and Fig. 3 in Arenou et al. 2018). As stated in Sect. 5.1 we limit our study to candidates within $30 \mathrm{pc}$ because the tidal radius may be higher (for example as we have not counted binaries, dust, etc..) and to look for objects that have been stripped off but continue to have the Hyades kinematics. Therefore, from the distribution of sources that passed the membership criteria, we counted the numbers of objects in three annuli (40-50 pc, 50-60 pc, and 60-70 pc) and divided by the volume, yielding numbers of contaminants in the range 3.84-7.59 $\times 10^{-4}$ per cubic parsec. Therefore, we predict $0.15-0.25,1.0-2.2,8.2-16.3$, and 34.0-67.3 contaminants among the $85,381,568$, and 710 sources in the 3.1, 3.1-9, 9-18, and $18-30 \mathrm{pc}$ volumes, respectively

We should add ten brown dwarfs to our luminosity function but we do not have Gaia magnitudes because they are too faint to be detected (Table 2; Sect. 4). We estimated their magnitudes with the equation in Sect. 4.1 (Smart et al. 2017b). We find 1, $1,4,1$, and 1 objects in the 16-17, 18-19, 19-20, 21-22, and 23-24 magnitude bins, respectively (red lines in Fig. 8), scaled to the volume and distance from the cluster centre. We note that there is only one brown dwarf in the $18-30 \mathrm{pc}$ annulus, all the others being within $14 \mathrm{pc}$.

Among the WDs, we have two in the $3.1 \mathrm{pc}$ core annulus (EGGR 36, EGGR 37) and another nine within the tidal radius (EGGR 29, HZ 14, LP 474-185, HG7-85, EGGR 316, HZ 7, HD 27483), including two known binaries V 471 Tau and HZ 9. Two other WDs (HZ4 and GD 52) lie between 14.9 and $16.0 \mathrm{pc}$. The remaining two WDs (GD 77 and G 74-15B) are 31-32 pc away from the cluster centre, which we reject as members of the cluster. None of the WDs have Gaia radial velocity.

We observe distinct shapes of the Hyades luminosity function in different regions of the cluster, from the core to the tidal radius, and the halo as previously reported by studies using HipPaRcos and ground-based data. In the central $3.1 \mathrm{pc}$, we observe two peaks at $M_{G}=5.0-6.0 \mathrm{mag}$ and 11-12 mag, corresponding to solar-type stars $\left(0.9 \pm 0.1 M_{\odot}\right)$ and M2-M4 dwarfs $\left(\sim 0.2-0.3 M_{\odot}\right)$ before it decreases sharply with only one brown dwarf below $M_{G}=16 \mathrm{mag}$ (HyaL5). The luminosity function increases smoothly until it peaks at $M_{G} \sim 12 \mathrm{mag}$ within the tidal radius and the brown dwarf bins are populated. Therefore, the lack of brown dwarfs in the core of the Hyades is likely of physical grounds because Gaia is most likely complete down to $0.072 M_{\odot}$ in magnitude space (with the biases described above). The peak of the luminosity function remains constant around 

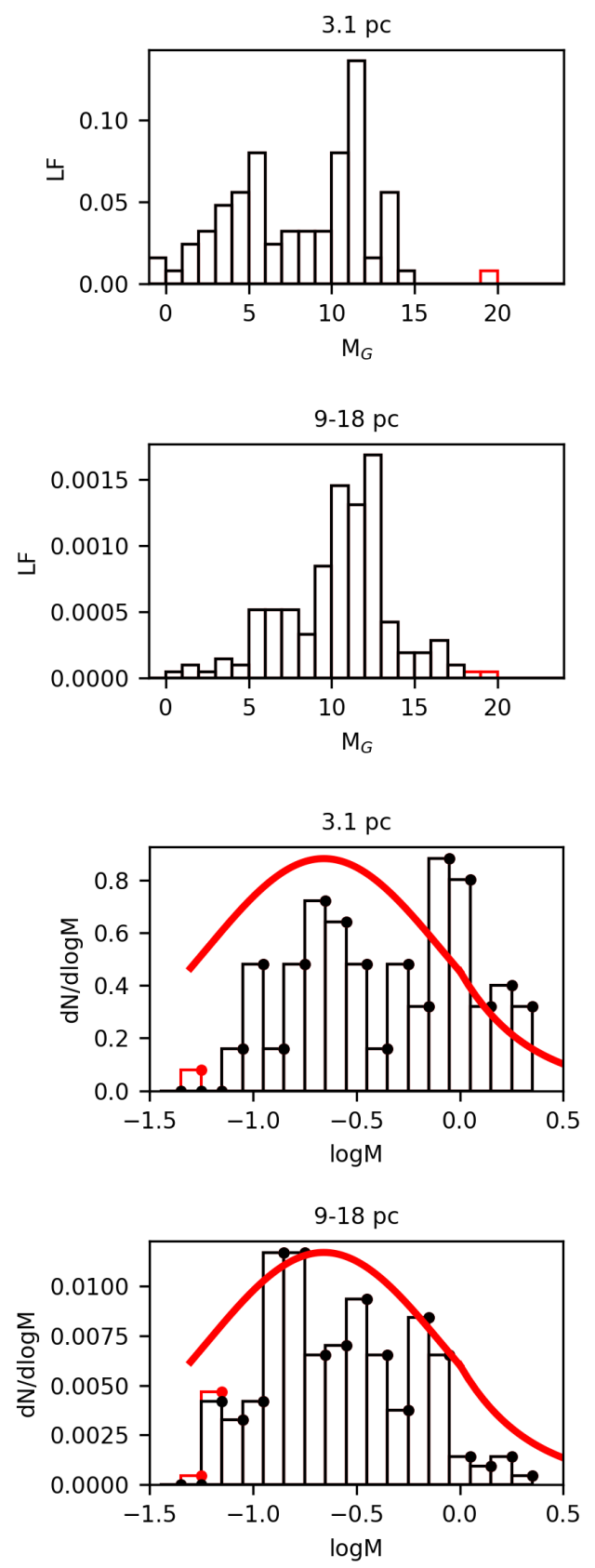
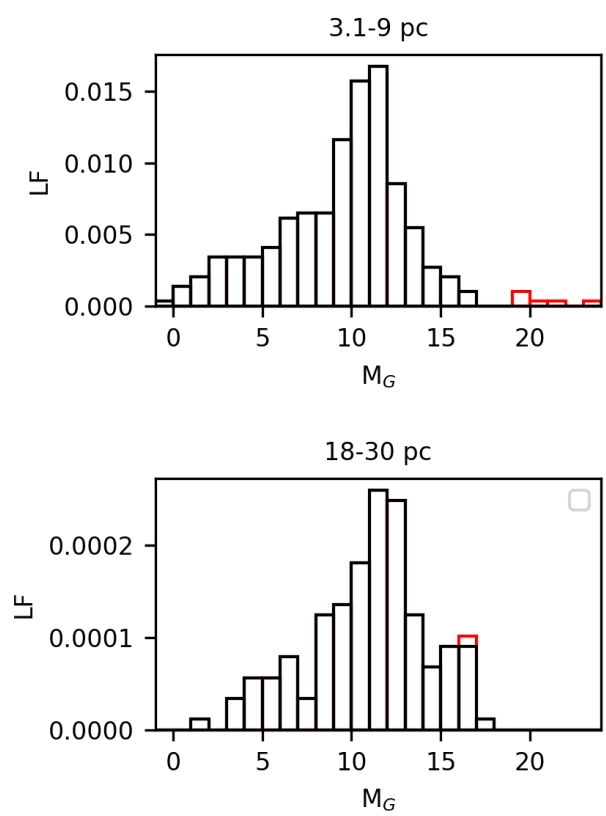

Fig. 8. Luminosity functions for radius intervals of $0-3.1 \mathrm{pc}, 3.1-9 \mathrm{pc}, 9-18 \mathrm{pc}$, and $18-30 \mathrm{pc}$ from the cluster centre scaled to a volume of one cubic parsec. We added the brown dwarfs undetected in Gaia DR2 in red.
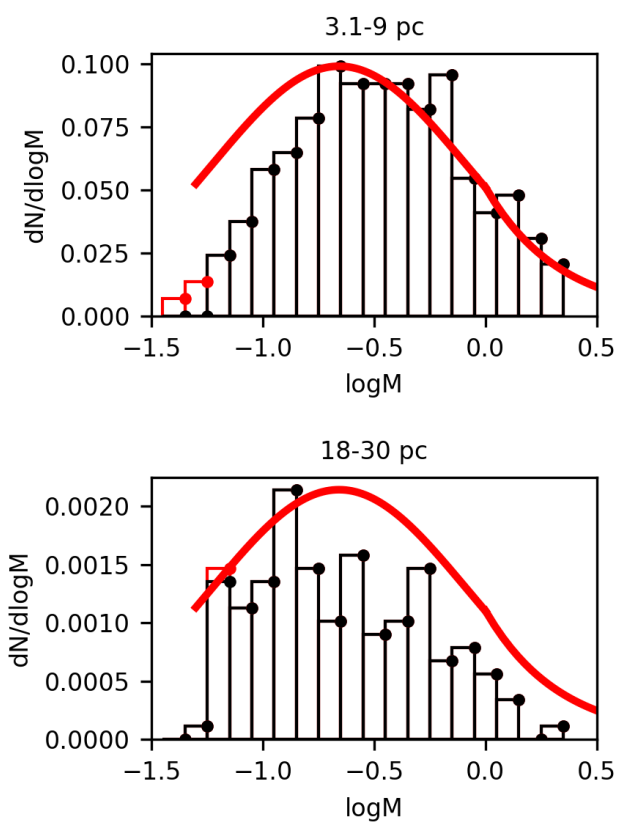

Fig. 9. Mass functions for annuli of 0 $3.1 \mathrm{pc}, 3.1-9 \mathrm{pc}, 9-18 \mathrm{pc}$, and $18-30 \mathrm{pc}$ from the cluster centre scaled to a volume of one cubic parsec and mass bins of 0.1 dex in logarithmic units assuming an age of 650 Myr. Overplotted with a thick red line is the lognormal form of the field mass function (Chabrier 2003) normalised to one and multiplied by the most populated bin in the volume considered.
$M_{G} \sim 12 \mathrm{mag}$ (M2-M4) beyond the core radius. Assuming magnitude as a proxy for mass we find the relative number per cubic parsec of high-mass stars $\left(\geq 1.4 M_{\odot}\right)$ in the $3.1-9$ pc annulus is 14.75 times lower than in the core. The number of highmass stars decreases by a factor of $\sim 30$ and $\sim 10$ in the next two annuli, while the number of solar-type stars $\left(0.7-1.3 M_{\odot}\right)$ and low-mass stars $\left(0.1-0.5 M_{\odot}\right)$ decrease by a factor of $14(5.4)$ and 10.6 (6.3), respectively. The relative density of low-mass stars to high-mass stars is 2.6 in the core radius but increases to 9.2 and 30.2 in the tidal radius and halo, respectively. The luminosity function increases steadily in the 9-18 pc region until it reaches a broader peak at $M_{G}=13 \mathrm{mag}$, one magnitude fainter than at closer radii, and remains so in the halo.

\subsection{Mass function}

To convert magnitudes into masses, we need a mass-luminosity relation over a wide mass range, from A-type stars down to the sub-stellar regime. To derive the most reliable present-day mass function, we would require eclipsing binaries with accurate masses and radii at the age of the Hyades. This information is not available despite recent advances thanks to Kepler/K2 which identified transiting planets orbiting members of the Hyades (Ciardi et al. 2018; David et al. 2016; Livingston et al. 2018; Mann et al. 2016, 2018). We selected both Hyades planet-host stars (EPIC 247589423 and EPIC 210490365) as kinematic candidates with distances from the cluster centre of $12.96 \mathrm{pc}$ and $4.45 \mathrm{pc}$ of the cluster, respectively. We remark that EPIC 247589423 lies beyond the tidal radius of the cluster but has a radial velocity from Gaia consistent with the cluster. Since we do not have an empirical calibration, we adopt a model-dependent mass-magnitude relation and note that all the results in this section follow from that. We considered two types of models at an age of $650 \mathrm{Myr}$ to convert observables into masses: the Padova isochrones (PARSEC v1.2S + COLIBRI PR16; Bressan et al. 2012; Marigo et al. 2013, 2008, 2017; 
Rosenfield et al. 2016) ${ }^{5}$ and the BT-Settl models (Allard et al. 2012; Baraffe et al. 2015) ${ }^{6}$. We also produced combined models for ages of $600 \mathrm{Myr}$ and $700 \mathrm{Myr}$.

We compared the masses predicted by the BT-Settl isochrones to the dynamical masses of field late-M and L dwarfs (Dupuy \& Liu 2017). We should bear in mind the difference in age between Hyades members $(650 \pm 100 \mathrm{Myr})$ and field dwarfs ( $>1$ Gyr). Based on the four candidates of Hogan et al. (2008) with optical spectral types recovered in our Gaia sample (Hya01, Hya03, Hya06, and Hya08), we would infer masses of $0.098 \pm 0.011$ and $0.085 \pm 0.010 M_{\odot}$ for M8-M8.5 and M9-L0.5 dwarfs (Dupuy \& Liu 2017) while the models predict masses of 0.077 and $0.065-0.068 M_{\odot}$. Considering the difference in ages and the range in dynamical masses, the agreement between observed and model-dependent masses is acceptable to proceed with the derivation of the mass function bearing in mind these caveats.

We opted to merge both models to cover the full range of masses. We kept the Padova and BT-Settl models above and below $1.4 M_{\odot}\left(M_{G} \sim 3.1 \mathrm{mag}\right)$, respectively. We find small differences between both models at $1.4 M_{\odot}: \log \left(L / L_{\odot}\right)=0.62$ vs 0.60 dex in luminosities, $6918 \mathrm{~K}$ vs $6724 \mathrm{~K}$ in effective temperatures, and $\log g=4.28$ vs 4.25 dex in gravities. We have now a mass-luminosity relation from $2.6 M_{\odot}$ down to $0.05 M_{\odot}$ equivalent to absolute $G$ magnitudes of $-2.78 \mathrm{mag}$ and $19.03 \mathrm{mag}$, respectively. The full magnitude range of apparent Gaia magnitudes (3-21 mag) is therefore covered as are brown dwarfs in the Hyades.

We plot the (system) mass function in Fig. 9 counting the number of objects per volume cubic parsec and per bins of 0.1 dex in logarithmic units of mass, assuming an age of $650 \mathrm{Myr}$. We obtained the masses for the brown dwarfs in a different manner because we have detection of lithium in absorption at $6707.8 \AA$ for some of them, placing their mass in the 0.05-0.06 $M_{\odot}$ range (Baraffe et al. 2015; Basri et al. 1996; Rebolo et al. 1992). We place Hya02 (M8.5) and Hya11 (L3.0) in the 0.06-0.07 $M_{\odot}$ mass bin because they have depleted their lithium, while the $\mathrm{T}$ dwarfs from Bouvier et al. (2008) have most likely masses below $0.05 M_{\odot}$. Therefore, we place two, seven, and two sub-stellar members in the 0.06-0.07, 0.05-0.06, and 0.04-0.05 $M_{\odot}$ intervals, respectively, in addition to all the Gaia members.

We plot the Hyades (system) mass function for four annuli $(3.1,3.1-9,9-18$, and 18-30 pc) in Fig. 9. We overplot the lognormal form of the field mass function (red line) from Chabrier (2003), normalised to the value of the most populated bin of objects in the volume into consideration. We observe that the Hyades mass function is not reproduced by the field mass function in any of the regions. In the core of the cluster, we observe an excess of high-mass stars $\left(\geq 1.4 M_{\odot}\right)$ with respect to the field while low-mass stars are under-represented. The mass function within the tidal radius is relatively well fit by the log-normal form of the field IMF, except for stars below $0.1 M_{\odot}$ and brown dwarfs. In the halo, the high-mass stars are clearly under-represented because their number is scarce while low-mass stars start to dominate The lack of brown dwarfs remain evident in all regions, which we attribute to the mass segregation and the incompleteness of previous ground-based surveys in the sub-stellar regime due to a combination of a lack of sensitivity and limited spatial coverage (Bouvier et al. 2008; Pérez-Garrido et al. 2017, 2018).

As stated above, the system mass function is not corrected for binaries, which we cannot do at this stage. The upcoming

\footnotetext{
5 http://stev.oapd.inaf.it/cgi-bin/cmd

6 https://phoenix.ens-lyon.fr/Grids/BT-Settl/

CIFIST2011_2015/ISOCHRONES/
}

release of Gaia will contribute to the correction but a wider spectrum of physical separations must be probed before a "resolved" mass function can be derived. To estimate the impact of binaries on the shape of the mass function, we have compared the system and resolved mass functions of the field (Fig. 1 and Eq. (17) + (18) in Chabrier 2003). Assuming that the multiplicity of Hyades members is comparable to the binary fractions in the solar neighbourhood, we should apply the multiplicative factors of $0.96,1.0,1.14,2.17$, and 3.33 to the numbers of members at masses of $2.0,1.0,0.5,0.3$, and $0.1 M_{\odot}$, respectively.

We infer a total mass of $61 M_{\odot}$ in 85 systems within a radius of $3.1 \mathrm{pc}$ from the cluster centre assuming an age of $650 \mathrm{Myr}$ and the models described earlier. The total mass is about 218 , 292, and $343 M_{\odot}$ in 381,568 , and 710 systems within 9,18 , and $30 \mathrm{pc}$ from the cluster centre, respectively. We find a slightly larger number of systems within the central 9 pc than Röser et al. (2011) with a smaller total mass (381 vs 364 systems and $218 M_{\odot}$ vs $\left.275 M_{\odot}\right)$. We find another $\sim 70 M_{\odot}$ in the halo $(9-18 \mathrm{pc}$ ), which is two-thirds of the one reported by Röser et al. (2011) but a similar additional mass budget in the $18-30 \mathrm{pc}$ annulus $\left(\sim 50 M_{\odot}\right.$ vs $\left.60 M_{\odot}\right)$. The most likely explanation is that the high astrometric precision of the Gaia data has cleaned up the contaminants in the halo of the cluster but possibly not fully beyond where we still expect significant contamination (lower right panel in Fig. 8). We caution that those total mass estimates do not consider multiple systems. Assuming a multiplicity fraction of $20-40 \%$ and an average mass of the secondary equal to two-thirds of the primary, the correction factor to apply to the aforementioned numbers would be of order of 16-27\%, which is not negligible at all. We inferred a tidal radius of 8.3 pc from Gaia DR2 alone, agree with Röser et al. (2011). Accounting for $20-40 \%$ binaries among Hyades members, the tidal radius of the cluster may increase to $10.5-13.5 \mathrm{pc}$ depending on the binary fraction and mass ratios. We also investigated the impact of the uncertainty on the age of the Hyades by calculating the total mass in each distance annulus for isochrones of $600 \mathrm{Myr}$ and $700 \mathrm{Myr}$, corresponding to about $10 \%$ uncertainty. At those ages, the isochrones are very similar over a wide range of masses, except above $1.8 M_{\odot}$ and below the hydrogen-burning limit. Both mass intervals contain a limited number of members. The impact is less than $1 \%$ on the total mass, therefore minimal, and much lower than the effect of multiplicity discussed above.

\section{Discussion: a 3D view of the Hyades}

\subsection{Distribution in space}

With the availability of accurate astrometry from Gaia DR2, we are now able to draw a 3D map in galactic coordinates for all bona-fide members identified from their kinematics. We depict the distribution of all 1764 member candidates in Fig. 10 but limited the plots to the central regions where we overplotted four circles in cyan representing the core $(3.1 \mathrm{pc})$, tidal $(9 \mathrm{pc})$, halo $(18 \mathrm{pc})$, and $30 \mathrm{pc}$ radius. We added in red in Fig. 10 the galactic coordinates of ten brown dwarfs discussed earlier for which we have ground-based parallaxes, except in the case of the two mid-L candidates identified by Schneider et al. (2017) where we included their photometric distances for completeness. These are the only two sources without parallaxes in our sample in addition to Hya04 which has no parallax in Gaia DR2.

The $(0,0,0)$ in galactic coordinates represent the position of the Sun and $(1,0,0)$ is a unit vector pointing to the galactic centre. We observe that the cluster shows a centrally-concentrated group of stars with two tails in the $\mathrm{X}$ and $\mathrm{Y}$ directions (Fig. 10). 

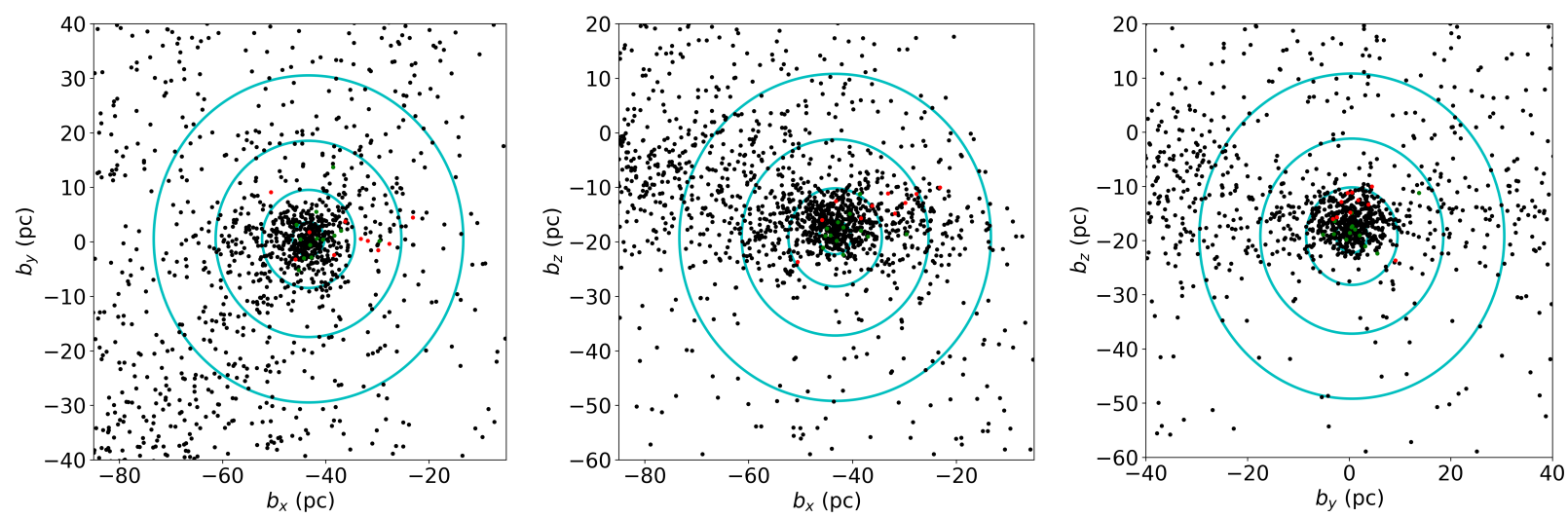

Fig. 10. 3D coordinates in space $\left(\boldsymbol{b}_{\mathrm{c}}\right.$ in pc) of the 1764 Hyades stellar (black) and ten sub-stellar (red) members with four different annuli from the cluster centre drawn in cyan: $3.1,9,18$, and $30 \mathrm{pc}$. White dwarfs are highlighted in green.
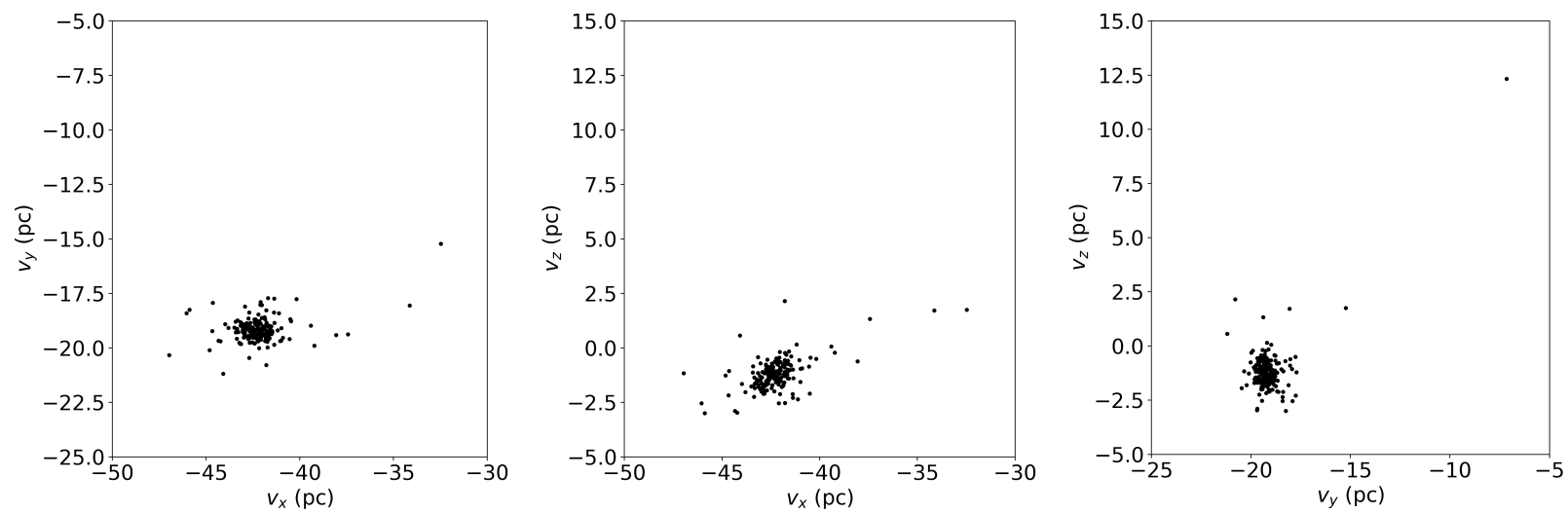

Fig. 11. 3D velocities of 192 Hyades members within $30 \mathrm{pc}$ with Gaia RVs. One object lies outside the limits of the $\left(v_{x}, v_{y}\right)$ plot with $v_{x}=-17.26 \mathrm{pc}$ (the outlier at the top-right on the right-hand side diagram).

The extension of the cluster in $\mathrm{Y}, \mathrm{Z}$ is consistent with the extension found in the Hipparcos data (Perryman et al. 1998). We refer to that work for a detailed interpretation of the possible causes for the spatial distribution of cluster members. The elongation of the cluster in the $\mathrm{X}$ direction is increasing dramatically from 3.1 to 9 pc from the cluster centre with the population in the tail to the Galactic anti-centre increasing quickly between $3.1,9$, and $18 \mathrm{pc}$. The overall extension of about $80 \mathrm{pc}$, from $-20 \mathrm{pc}$ to $-100 \mathrm{pc}$ is also consistent with the results of HIPPARCos (Perryman et al. 1998) but is now homogeneously distributed with the presence of a tail towards negative values of $b_{x}$. We note that the cluster appears also elongated in the $\mathrm{Y}$ direction beyond $18 \mathrm{pc}$, in the region of the halo of the cluster where we might identify a mix of members of the cluster and the Hyades moving group (Boss 1908; Eggen 1958; Famaey et al. 2007; Zuckerman \& Song 2004).

In Fig. 11, we plot the 3D distribution of galactic velocities $\left(\boldsymbol{b}_{\mathrm{c}}\right.$ in $\mathrm{km} \mathrm{s}^{-1}$ ) of the 192 Hyades members at distances less than $30 \mathrm{pc}$ from the cluster centre and Gaia radial velocities $(G=5.6-$ $13.6 \mathrm{mag}$ ). The size of the sample corresponds approximately to the size of the Hipparcos sample but with more accurate radial velocities. We observe an extension along the $X$-axis with a small sub-group of objects towards negative $v_{x}$. We confirm the conclusions drawn in Perryman et al. (1998).

\subsection{Mass segregation}

The differences seen in the luminosity function in different annuli from the cluster centre clearly indicate a mass segregation in the Hyades (Fig. 8), in fact extensively discussed in the literature (e.g. Bouvier et al. 2008; Perryman et al. 1998; Röser et al. 2011) and reproduced through numerical simulations by the loss of the lowest mass members over a timescale of a few $100 \mathrm{Myr}$ (de La Fuente Marcos 1995; Kroupa 1995; Terlevich 1987).

In Fig. 12 we display the distance from the centre of the cluster as a function of the Gaia magnitude $G$ for the 3.1, 9, 18, and $30 \mathrm{pc}$ radii from the cluster centre. We overplot the Hyades brown dwarfs in red. In these diagrams, as we can assume the objects are of the similar age, composition and distance then magnitude will act as a proxy for mass. We can clearly see the equal number of stars at different masses within the central $\sim 3 \mathrm{pc}$ whereas low-mass stars become more numerous beyond 3 pc. At distances larger than 6-7 pc, the number of high-mass stars become negligible (Fig. 12). We observe a possible bias of brown dwarf members on the side towards us, suggesting that we are more complete nearby than the other side, which can be a consequence of the depth of photometric surveys. These plots represent another proof of the mass segregation present in the Hyades. We observe that one of the known brown dwarfs lies at $2.6 \mathrm{pc}$ from the cluster (red dots in Fig. 10). Most of them lie within the tidal radius and the furthest at about $20 \mathrm{pc}$. Their distribution seems to match the distribution of M-type members.

To illustrate the effect of segregation in a more quantitative manner, we compute the ratio $R$ of stars to brown dwarfs as defined by Andersen et al. (2008): the sum of all objects in the interval $0.08-1.0 M_{\odot}$ is divided by the number of brown dwarfs $\left(0.03-0.08 M_{\odot}\right)$. We emphasise that the bin of brown dwarfs is yet incomplete in the Hyades because the coolest members 


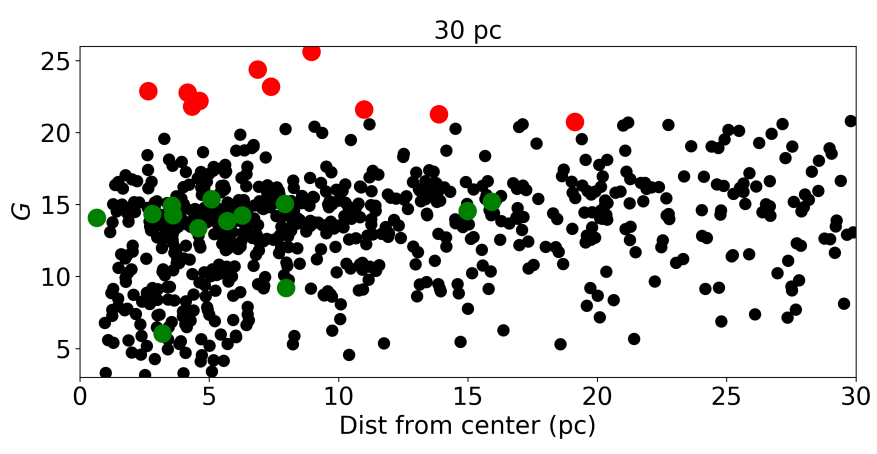

Fig. 12. Distance from the cluster centre in $3 \mathrm{D}$ space vs $G$ magnitude for Hyades members selected kinematically up to a radius of $30 \mathrm{pc}$. We overplotted brown dwarfs with ground-based parallaxes and white dwarfs as red and green dots, respectively.

are early-T dwarfs with model-dependent masses of the order of $0.05 M_{\odot}$ (Bouvier et al. 2008) and confirmed L-type brown dwarf with lithium in absorption at $6707.8 \AA$ and masses in the range $0.05-0.06 M_{\odot}$ (Lodieu et al. 2018; Martín et al. 2018). The $0.03-0.05 M_{\odot}$ bin remains currently unexplored in the Hyades cluster; thus all number are upper limits. As described in Sect. 4 we have confirmed spectroscopically, as well as through groundbased parallaxes, the presence of eight brown dwarfs in the cluster to which we should add the two L5+L6 dwarfs from Schneider et al. (2017). Among these ten brown dwarfs, three are located in the core, four in the $3.1-9 \mathrm{pc}$ region, two in the 9-18 pc annulus, and one even farther. We will take into this fact into account in the following computations.

In the central $3.1 \mathrm{pc}$, we find an upper limit of $\mathrm{R}=62$ with the lack of object with a model-dependent mass below $0.08 M_{\odot}$ to which we added the only brown dwarf HyaL5. This ratio decreases with larger radii, going from $\mathrm{R}=22.1$ to 18.4 , and 14.6 at 9,18 , and $30 \mathrm{pc}$ from the cluster centre, respectively (after correction for the numbers of brown dwarfs). However, we argue that the search for sub-stellar members is incomplete beyond the tidal radius either because previous surveys ignored those regions or were not sensitive enough. Therefore, the last two ratios are clearly upper limit rather than exact values. Nonetheless, the evolution of the ratio as a function of distance from the cluster centre is another proof of the mass segregation in the Hyades cluster even though we shall keep in mind that these values are upper limits. Those ratios should be compared to the values of 3.3-8.5 and 4.9 derived for star-forming regions (age $=1-2 \mathrm{Myr}$ ) and the Pleiades $(120 \mathrm{Myr})$, respectively (Andersen et al. 2008), demonstrating the loss or incompleteness of sub-stellar members in the central part of the Hyades.

We extrapolated the current sample of stars within $8 \mathrm{pc}$ of the Sun to investigate the numbers of solar-type, low-mass, and brown dwarfs that may have been lost due to dynamical evolution. This sample is well reproduced by field mass functions (Chabrier 2003; Kroupa et al. 2013). From Fig. 11 of Kirkpatrick et al. (2012), the compilation of all known members within 8 pc of the Sun gives 11 WDs, 4 A, 2 F, 8 G, 29 K, 157 $\mathrm{M}$, and $3 \mathrm{~L}$ dwarfs with a lower numbers of $22 \mathrm{~T}$ dwarfs. We assume that all F-type members of the Hyades have been identified because their lifetime is larger than the age of the cluster, which might not be the case for all A stars. However, if we consider that all A-type stars have been identified, the extrapolations of the numbers of GKMLT dwarfs should be decreased by a factor of two approximately because we find roughly the same numbers of A and F stars. The same remark would apply if we consider the $G$ stars because we find about the same numbers of $F$ and $G$ dwarfs
Table 7. Observed numbers of Hyades members per spectral type as a function of distance from the cluster centre and expected numbers (in parenthesis) extrapolating the numbers of KMLT dwarfs from the census of the 8 pc volume-limited sample (Kirkpatrick et al. 2012) assuming that we identified all F-type members.

\begin{tabular}{lccccccc}
\hline \hline dist & $A$ & $F$ & $G$ & $K$ & $M$ & $L$ & $T$ \\
\hline 3.1 & 8 & 11 & $11(44)$ & $12(160)$ & $40(864)$ & $1(17)$ & $0(\geq 121)$ \\
9.0 & 25 & 29 & $29(112)$ & $75(406)$ & $209(2277)$ & $5(44)$ & $2(\geq 319)$ \\
18.0 & 29 & 31 & $42(124)$ & $110(450)$ & $330(2434)$ & $7(47)$ & $2(\geq 341)$ \\
30.0 & 31 & 36 & $51(144)$ & $133(522)$ & $419(2826)$ & $8(54)$ & $2(\geq 396)$ \\
\hline
\end{tabular}

in all annuli, implying that our extrapolations would decrease by a factor of approximately four. We note that 71 Tauri (F0V) is missing from our final catalogue (Sect. 5.2.4), hence affecting our estimates by at most $\sim 5 \%$. The other bright object missing $\left(\Theta^{1}\right.$ Tauri) is a giant, hence, does not affect our estimates. These differences could be due to several effects, among which mass segregation or stellar formation because field stars have an average age of 24 Gyr several times the age of the Hyades during which the star formation may have changed.

We list the numbers of observed dwarfs as a function of spectral type and distance from the cluster centre in Table 7 and give the extrapolated numbers in parenthesis, assuming the above conditions. We observe that the cluster has retained about $25 \%$ and $18 \%$ of its $\mathrm{G}$ and $\mathrm{K}$ dwarfs within its tidal radius and may have lost $90 \%$ of its $\mathrm{M}$ dwarfs. Numerical simulations of the dynamical evolution of clusters by Adams \& Myers (2001) suggest that a $650 \mathrm{Myr}$-old cluster may have lost $\sim 65 \%$ and $70-90 \%$ of its initial stellar and sub-stellar populations, fairly consistent with our analysis. The numbers of $\mathrm{L}$ and $\mathrm{T}$ dwarfs in the Hyades are quite low, which may be due to dynamical evolution but also to the lack of sensitivity and limited areal searches of previous studies (e.g. Bouvier et al. 2008; Hogan et al. 2008). We find five $\mathrm{L}$ dwarfs and two $\mathrm{T}$ dwarfs within the tidal radius, while we should have 44 and more than 319 based on the sample of $\mathrm{L}$ and $\mathrm{T}$ dwarfs within $8 \mathrm{pc}$ of the Sun. The upcoming Large Synoptic Survey telescope (LSST) might shed some light on this issue (Ivezic et al. 2008).

\section{Conclusions}

We presented updated parameters for the Hyades cluster as well as a revised census of member candidates up to $30 \mathrm{pc}$ from the cluster centre in the $2.50-0.04 M_{\odot}$ mass interval. The sample within the tidal radius of the cluster is $99 \%$ complete while the $18 \mathrm{pc}$ and $30 \mathrm{pc}$ samples might suffer from a level of contamination of $\leq 3 \%$ and $\sim 5-10 \%$, respectively. We combined the Gaia DR2 astrometry with ground-based parallaxes from the Liverpool telescope to produce the first 3D map of the cluster ${ }^{7}$.

We summarise the main results of our work:

- we derive a mean distance of $47.03 \pm 0.20 \mathrm{pc}$ for the cluster and a mean velocity of $46.38 \pm 0.12 \mathrm{~km} \mathrm{~s}^{-1}$ from members within the tidal radius

- we present ground-based parallaxes derived with the IO:I infrared camera on the $2 \mathrm{~m}$ robotic Liverpool telescope for eight of the ten known Hyades brown dwarfs.

- we identified a total of $85,381,568$, and 710 astrometric members within 3.1 (core radius), 9 (tidal radius), 18 (halo), and $30 \mathrm{pc}$ from the centre of the Hyades with

\footnotetext{
7 A movie is available at https://www.dropbox.com/s/ 19m4q08eenkzq8z/animation_Hyades_30pc.mp4?dl=0
} 
the implementation of the kinematic method described by Perryman et al. (1998). This sequence of Hyades members at $650 \mathrm{Myr}$ constitutes a benchmark in colour-magnitude diagrams involving optical to infrared magnitudes.

- we confirm the membership of previously-known white dwarfs and assess the membership of other possible pre-DR2 white dwarf candidates. We derive an age of $640_{-49}^{+67} \mathrm{Myr}$ from the nine single white dwarfs by comparing their Gaia photometry with state-of-the-art models, agree with the age derived from the lithium depletion boundary method.

- we derive the luminosity and mass functions of the Hyades in the $G=3-26$ mag range translating into masses in the 2.5$0.04 M_{\odot}$ interval. The shapes vary as a function of the distances from the centre.

- we derive 3D positions for all stellar and sub-stellar members and show a 3D map of the Hyades. The cluster centre is identified by $-43.83 \pm 0.18,+0.42 \pm 0.11,-17.05 \pm 0.09 \mathrm{pc}$ in $b_{x}$, $b_{y}$, and $b_{z}$, respectively.

- we observe a spatially-concentrated distribution of stellar members in 3D space with the extension of members towards the direction of the Galactic centre in velocity space and along the $\left(b_{x}, b_{y}\right)$ axis in coordinate space, as previously reported in studies exploiting HIPPARcos and ground-based data.

- we find that the cluster has clearly suffered mass segregation with the low-mass members being on average further away from the centre than high-mass and solar-type members.

Our study has ignored multiplicity over the full mass range. The next Gaia release should include information on binaries with some preliminary orbits to study the multiplicity s a function of mass and improve the mass determinations of the Hyades members to derive a more accurate mass function.

Finally, spectroscopic follow-up at medium-resolution is required to infer radial velocities of Gaia member candidates fainter than $G \sim 13.5 \mathrm{mag}$ and clean the astrometric sequence beyond the tidal radius.

Acknowledgements. NL and APG were financially supported by the Spanish Ministry of Economy and Competitiveness (MINECO) under the grants AYA2015-69350-C3-2-P and AYA2015-69350-C3-3-P. The authors would like to thank Jonathan Gagné and Jos de Bruijne for useful discussions during this work as well as Pierre Bergeron for having made available, before publication, the WD cooling tracks complete with Gaia magnitudes. This research has made use of the Simbad and Vizier databases, operated at the centrede Données Astronomiques de Strasbourg (CDS), and of NASA's Astrophysics Data System Bibliographic Services (ADS). This research has also made use of some of the tools developed as part of the Virtual Observatory. This work has made use of data from the European Space Agency (ESA) mission Gaia (https://www. cosmos.esa.int/gaia), processed by the Gaia Data Processing and Analysis Consortium (DPAC, https://www.cosmos.esa.int/web/gaia/dpac/ consortium). Funding for the DPAC has been provided by national institutions participating in the Gaia Multilateral Agreement and in particular the support of the Agenzia Spaziale Italiana under contract I/058/10/0. The Liverpoo Telescope is operated on the island of La Palma by Liverpool John Moores University in the Spanish Observatorio del Roque de los Muchachos of the Instituto de Astrofísica de Canarias with financial support from the UK Science and Technology Facilities Council. Funding for the Sloan Digital Sky Survey IV has been provided by the Alfred P. Sloan Foundation, the U.S. Department of Energy Office of Science, and the Participating Institutions. SDSS-IV acknowledges support and resources from the Center for High-Performance Computing at the University of Utah. The SDSS web site is www.sdss.org. SDSS-IV is managed by the Astrophysical Research Consortium for the Participating Institutions of the SDSS Collaboration including the Brazilian Participation Group, the Carnegie Institution for Science, Carnegie Mellon University, the Chilean Participation Group, the French Participation Group, HarvardSmithsonian Center for Astrophysics, Instituto de Astrofísica de Canarias, The Johns Hopkins University, Kavli Institute for the Physics and Mathematics of the Universe (IPMU) / University of Tokyo, Lawrence Berkeley National Laboratory, Leibniz Institut für Astrophysik Potsdam (AIP), Max-Planck-Institut für Astronomie (MPIA Heidelberg), Max-Planck-Institut für Astrophysik (MPA
Garching), Max-Planck-Institut für Extraterrestrische Physik (MPE), National Astronomical Observatories of China, New Mexico State University, New York University, University of Notre Dame, Observatário Nacional / MCTI, The Ohio State University, Pennsylvania State University, Shanghai Astronomical Observatory, United Kingdom Participation Group, Universidad Nacional Autónoma de México, University of Arizona, University of Colorado Boulder, University of Oxford, University of Portsmouth, University of Utah, University of Virginia, University of Washington, University of Wisconsin, Vanderbilt University, and Yale University. This publication makes use of data products from the Two Micron All Sky Survey, which is a joint project of the University of Massachusetts and the Infrared Processing and Analysis Center/California Institute of Technology, funded by the National Aeronautics and Space Administration and the National Science Foundation. The UKIDSS project is defined in Lawrence et al. (2007). UKIDSS uses the UKIRT Wide Field Camera Casali et al. (2007; WFCAM). The photometric system is described in Hewett et al. (2006), and the calibration is described in Hodgkin et al. (2009). The pipeline processing and science archive are described in Irwin et al. (in prep.) and Hambly et al. (2008). This publication makes use of data products from the Wide-field Infrared Survey Explorer, which is a joint project of the University of California, Los Angeles, and the Jet Propulsion Laboratory/ California Institute of Technology, and NEOWISE, which is a project of the Jet Propulsion Laboratory/California Institute of Technology. WISE and NEOWISE are funded by the National Aeronautics and Space Administration. The PanSTARRS1 Surveys (PS1) and the PS1 public science archive have been made possible through contributions by the Institute for Astronomy, the University of Hawaii, the Pan-STARRS Project Office, the Max-Planck Society and its participating institutes, the Max Planck Institute for Astronomy, Heidelberg and the Max Planck Institute for Extraterrestrial Physics, Garching, The Johns Hopkins University, Durham University, the University of Edinburgh, the Queen's University Belfast, the Harvard-Smithsonian Center for Astrophysics, the Las Cumbres Observatory Global Telescope Network Incorporated, the National Central University of Taiwan, the Space Telescope Science Institute, the National Aeronautics and Space Administration under Grant No. NNX08AR22G issued through the Planetary Science Division of the NASA Science Mission Directorate, the National Science Foundation Grant No. AST-1238877, the University of Maryland, Eotvos Lorand University (ELTE), the Los Alamos National Laboratory, and the Gordon and Betty Moore Foundation.

\section{References}

Abolfathi, B., Aguado, D. S., Aguilar, G., et al. 2018, ApJS, 235, 42 Adams, F. C., \& Myers, P. C. 2001, ApJ, 553, 744

Allard, F., Homeier, D., \& Freytag, B. 2012, Roy. Soc. London Philos. Trans. Ser. A, 370, 2765

Andersen, M., Meyer, M. R., Greissl, J., \& Aversa, A. 2008, ApJ, 683, L183

Arenou, F., Luri, X., Babusiaux, C., et al. 2018, A\&A, 616, A17

Artigau, É., Gagné, J., Faherty, J., et al. 2015, ApJ, 806, 254

Baraffe, I., Homeier, D., Allard, F., \& Chabrier, G. 2015, A\&A, 577, A42

Barnsley, R. M., Jermak, H. E., Steele, I. A., et al. 2016, J. Astron. Telesc. Instrum. Syst., 2, 015002

Barrado y Navascués, D., Bouvier, J., Stauffer, J. R., Lodieu, N., \& McCaughrean, M. J. 2002, A\&A, 395, 813

Basri, G., Marcy, G. W., \& Graham, J. R. 1996, ApJ, 458, 600

Bergeron, P., Wesemael, F., Dufour, P., et al. 2011, ApJ, 737, 28

Boesgaard, A. M., \& Friel, E. D. 1990, ApJ, 351, 467

Boss, L. J. 1908, AJ, 26, 31

Boucher, A., Lafrenière, D., Gagné, J., et al. 2016, ApJ, 832, 50

Bouvier, J., Stauffer, J. R., Martín, E. L., et al. 1998, A\&A, 336, 490

Bouvier, J., Kendall, T., Meeus, G., et al. 2008, A\&A, 481, 661

Brandt, T. D., \& Huang, C. X. 2015, ApJ, 807, 58

Bressan, A., Marigo, P., Girardi, L., et al. 2012, MNRAS, 427, 127

Bryja, C., Humphreys, R. M., \& Jones, T. J. 1994, AJ, 107, 246

Casali, M., Adamson, A., Alves de Oliveira, C., et al. 2007, A\&A, 467, 777

Casewell, S. L., Littlefair, S. P., Burleigh, M. R., \& Roy, M. 2014, ArXiv e-prints [arXiv:1404.3970]

Cayrel de Strobel, G., Crifo, F., \& Lebreton, Y. 1997, in Hipparcos - Venice '97, eds. R. M. Bonnet, E. Høg, P. L. Bernacca, et al., ESA SP, 402, 687 Chabrier, G. 2003, PASP, 115, 763

Chambers, K. C., Magnier, E. A., Metcalfe, N., et al. 2016, ArXiv e-prints [arXiv:1612.05560]

Ciardi, D. R., Crossfield, I. J. M., Feinstein, A. D., et al. 2018, AJ, 155, 10

Cutri, R. M., Skrutskie, M. F., van Dyk, S., et al. 2003, 2MASS All Sky Catalog of point sources, 2246

Cutri, R. M., et al. 2014, VizieR Online Data Catalog: II/328

David, T. J., Conroy, K. E., Hillenbrand, L. A., et al. 2016, AJ, 151, 112

de Bruijne, J. H. J., Hoogerwerf, R., \& de Zeeuw, P. T. 2001, A\&A, 367, 111

De Gennaro, S., von Hippel, T., Jefferys, W. H., et al. 2009, ApJ, 696, 12 
de La Fuente Marcos, R. 1995, A\&A, 301, 407

Dias, W. S., Alessi, B. S., Moitinho, A., \& Lépine, J. R. D. 2002, A\&A, 389, 871

Dias, W. S., Assafin, M., Flório, V., Alessi, B. S., \& Líbero, V. 2006, A\&A, 446, 949

Dias, W. S., Monteiro, H., Caetano, T. C., et al. 2014, A\&A, 564, A79

Dias, W. S., Monteiro, H., \& Assafin, M. 2018, MNRAS, 478, 5184

Dobbie, P. D., Kenyon, F., Jameson, R. F., et al. 2002, MNRAS, 329, 543

Duchêne, G., Bouvier, J., Moraux, E., et al. 2013, A\&A, 555, A137

Dupuy, T. J., \& Liu, M. C. 2017, ApJS, 231, 15

Eggen, O. J. 1958, MNRAS, 118, 65

Eggen, O. J. 1969, ApJ, 158, 1109

Eggen, O. J. 1998, AJ, 116, 284

El-Badry, K., Rix, H.-W., \& Weisz, D. R. 2018, ApJ, 860, L17

Famaey, B., Pont, F., Luri, X., et al. 2007, A\&A, 461, 957

Gagné, J., Lafrenière, D., Doyon, R., Malo, L., \& Artigau, É. 2014, ApJ, 783, 121

Gagné, J., Lafrenière, D., Doyon, R., Malo, L., \& Artigau, É. 2015a, ApJ, 798, 73

Gagné, J., Faherty, J. K., Cruz, K. L., et al. 2015b, ApJS, 219, 33

Gagné, J., Faherty, J. K., Mamajek, E. E., et al. 2017, ApJS, 228, 18

Gagné, J., Mamajek, E. E., Malo, L., et al. 2018a, ApJ, 856, 23

Gagné, J., Roy-Loubier, O., Faherty, J. K., Doyon, R., \& Malo, L. 2018b, ApJ, 860,43

Gaia Collaboration (Prusti, T., et al.) 2016, A\&A, 595, A1

Gaia Collaboration (van Leeuwen, F., et al.) 2017, A\&A, 601, A19

Gaia Collaboration (Babusiaux, C., et al.) 2018a, A\&A, 616, A10

Gaia Collaboration (Brown, A. G. A., et al.) 2018b, A\&A, 616, A1

Gebran, M., Vick, M., Monier, R., \& Fossati, L. 2010, A\&A, 523, A71

Gizis, J. E., Reid, I. N., \& Monet, D. G. 1999, AJ, 118, 997

Goldman, B., Röser, S., Schilbach, E., et al. 2013, A\&A, 559, A43

Grenon, M. 2000, IAU Joint Discuss., 13

Gunn, J. E., Griffin, R. F., Griffin, R. E. M., \& Zimmerman, B. A. 1988, AJ, 96, 198

Hambly, N. C., Collins, R. S., Cross, N. J. G., et al. 2008, MNRAS, 384, 637

Hanson, R. B. 1975, AJ, 80, 379

Hewett, P. C., Warren, S. J., Leggett, S. K., \& Hodgkin, S. T. 2006, MNRAS, 367,454

Hodgkin, S. T., Irwin, M. J., Hewett, P. C., \& Warren, S. J. 2009, MNRAS, 394, 675

Høg, E., Fabricius, C., Makarov, V. V., et al. 2000, A\&A, 355, L27

Hogan, E., Jameson, R. F., Casewell, S. L., Osbourne, S. L., \& Hambly, N. C. 2008, MNRAS, 388, 495

Ivezic, Z., Axelrod, T., Brandt, W. N., et al. 2008, Serb. Astron. J., 176, 1

Jones, D. H. P. 1971, MNRAS, 152, 231

Kaiser, N., Aussel, H., Burke, B. E., et al. 2002, in Survey and Other Telescope Technologies and Discoveries, eds. J. A. Tyson, \& S. Wolff, SPIE Conf. Ser., 4836, 154

Keenan, P. C., \& McNeil, R. C. 1989, ApJS, 71, 245

Kirkpatrick, J. D., Gelino, C. R., Cushing, M. C., et al. 2012, ApJ, 753, 156

Kroupa, P. 1995, MNRAS, 277, 1522

Kroupa, P., Weidner, C., Pflamm-Altenburg, J., et al. 2013, in The Stellar and Sub-Stellar Initial Mass Function of Simple and Composite Populations, eds. T. D. Oswalt, \& G. Gilmore, 115

Lada, C. J., \& Lada, E. A. 2003, ARA\&A, 41, 57

Lawrence, A., Warren, S. J., Almaini, O., et al. 2007, MNRAS, 379, 1599

Lebreton, Y., Fernandes, J., \& Lejeune, T. 2001, A\&A, 374, 540

Leggett, S. K., \& Hawkins, M. R. S. 1989, MNRAS, 238, 145

Leggett, S. K., Harris, H. C., \& Dahn, C. C. 1994, AJ, 108, 944

Lindegren, L., Hernández, J., Bombrun, A., et al. 2018, A\&A, 616, A2

Livingston, J. H., Dai, F., Hirano, T., et al. 2018, AJ, 155, 115

Lodieu, N., McCaughrean, M. J., Barrado Y Navascués, D., Bouvier, J., \& Stauffer, J. R. 2005, A\&A, 436, 853

Lodieu, N., Boudreault, S., \& Béjar, V. J. S. 2014a, MNRAS, 445, 3908

Lodieu, N., Pérez-Garrido, A., Béjar, V. J. S., et al. 2014b, A\&A, 569, A120

Lodieu, N., Rebolo, R., \& Pérez-Garrido, A. 2018, A\&A, 615, L12

Luri, X., Brown, A. G. A., Sarro, L. M., et al. 2018, A\&A, 616, A9
Maeder, A., \& Mermilliod, J. C. 1981, A\&A, 93, 136

Malo, L., Doyon, R., Lafrenière, D., et al. 2013, ApJ, 762, 88

Malo, L., Artigau, É., Doyon, R., et al. 2014a, ApJ, 788, 81

Malo, L., Doyon, R., Feiden, G. A., et al. 2014b, ApJ, 792, 37

Mann, A. W., Gaidos, E., Mace, G. N., et al. 2016, ApJ, 818, 46

Mann, A. W., Vanderburg, A., Rizzuto, A. C., et al. 2018, AJ, 155, 4

Marigo, P., Girardi, L., Bressan, A., et al. 2008, A\&A, 482, 883

Marigo, P., Bressan, A., Nanni, A., Girardi, L., \& Pumo, M. L. 2013, MNRAS, 434,488

Marigo, P., Girardi, L., Bressan, A., et al. 2017, ApJ, 835, 77

Martín, E. L., Lodieu, N., Pavlenko, Y., \& Béjar, V. J. S. 2018, ApJ, 856, 40

Mazzei, P., \& Pigatto, L. 1988, A\&A, 193, 148

Melnikov, S., \& Eislöffel, J. 2018, A\&A, 611, A34

Mermilliod, J.-C. 1981, A\&A, 97, 235

Mermilliod, J.-C., Mayor, M., \& Udry, S. 2009, A\&A, 498, 949

Moraux, E., Bouvier, J., \& Stauffer, J. R. 2001, A\&A, 367, 211

Newton, E. R., Charbonneau, D., Irwin, J., \& Mann, A. W. 2015, ApJ, 800, 85

Pérez-Garrido, A., Lodieu, N., \& Rebolo, R. 2017, A\&A, 599, A78

Pérez-Garrido, A., Lodieu, N., Rebolo, R., \& Chinchilla, P. 2018, A\&A, 620, A130

Perryman, M. A. C., Lindegren, L., Kovalevsky, J., et al. 1997, A\&A, 323, L49

Perryman, M. A. C., Brown, A. G. A., Lebreton, Y., et al. 1998, A\&A, 331, 81

Rebolo, R., Martín, E. L., \& Magazzù, A. 1992, ApJ, 389, L83

Reid, N. 1992, MNRAS, 257, 257

Reid, N. 1993, MNRAS, 265, 785

Reid, I. N., \& Gizis, J. E. 1997, AJ, 114, 1992

Reid, I. N., \& Hawley, S. L. 1999, AJ, 117, 343

Reid, I. N., \& Mahoney, S. 2000, MNRAS, 316, 827

Reid, I. N., Kirkpatrick, J. D., Liebert, J., et al. 1999, ApJ, 521, 613

Reino, S., de Bruijne, J., Zari, E., d'Antona, F., \& Ventura, P. 2018, MNRAS, 477, 3197

Rosenfield, P., Marigo, P., Girardi, L., et al. 2016, ApJ, 822, 73

Röser, S., Demleitner, M., \& Schilbach, E. 2010, AJ, 139, 2440

Röser, S., Schilbach, E., Piskunov, A. E., Kharchenko, N. V., \& Scholz, R.-D. 2011, A\&A, 531, A92

Salaris, M., \& Bedin, L. R. 2018, MNRAS, 480, 3170

Schilbach, E., \& Röser, S. 2012, A\&A, 537, A129

Schneider, A. C., Windsor, J., Cushing, M. C., Kirkpatrick, J. D., \& Shkolnik, E. L. 2017, AJ, 153, 196

Skrutskie, M. F., Cutri, R. M., Stiening, R., et al. 2006, AJ, 131, 1163

Smart, R. L., Lattanzi, M. G., Bucciarelli, B., et al. 2003, A\&A, 404, 317

Smart, R. L., Ioannidis, G., Jones, H. R. A., Bucciarelli, B., \& Lattanzi, M. G. 2010, A\&A, 514, A84

Smart, R. L., Apai, D., Kirkpatrick, J. D., et al. 2017a, MNRAS, 468, 3764

Smart, R. L., Marocco, F., Caballero, J. A., et al. 2017b, MNRAS, 469, 401

Soderblom, D. R., Jones, B. F., Stauffer, J. R., \& Chaboyer, B. 1995, AJ, 110, 729

Stauffer, J. R., Liebert, J., Giampapa, M., et al. 1994, AJ, 108, 160

Stauffer, J. R., Liebert, J., \& Giampapa, M. 1995, AJ, 109, 298

Steele, I. A., Smith, R. J., Rees, P. C., et al. 2004, in Ground-based Telescopes, ed. J. M. Oschmann, Jr., Proc. SPIE, 5489, 679

Tabernero, H. M., Montes, D., \& González Hernández, J. I. 2012, A\&A, 547, A13

Tang, J., Bressan, A., Rosenfield, P., et al. 2014, MNRAS, 445, 4287

Taylor, B. J. 2006a, AJ, 132, 2453

Taylor, M. B. 2006b, in Astronomical Data Analysis Software and Systems XV, eds. C. Gabriel, C. Arviset, D. Ponz, \& S. Enrique, ASP Conf. Ser., 351, 666 Terlevich, E. 1987, MNRAS, 224, 193

Tody, D. 1986, in SPIE Conf. Ser., ed. D. L. Crawford, 627, 733

Tody, D. 1993, in Astronomical Data Analysis Software and Systems II, eds. R. J. Hanisch, R. J. V. Brissenden, \& J. Barnes, ASP Conf. Ser., 52, 173

Tremblay, P.-E., Schilbach, E., Röser, S., et al. 2012, A\&A, 547, A99

von Hippel, T. 1998, AJ, 115, 1536

Weidemann, V., Jordan, S., Iben, Jr., I., \& Casertano, S. 1992, AJ, 104, 1876

White, R. J., Gabor, J. M., \& Hillenbrand, L. A. 2007, AJ, 133, 2524

Wright, E. L., Eisenhardt, P. R. M., Mainzer, A. K., et al. 2010, AJ, 140, 1868

Zuckerman, B., \& Song, I. 2004, ARA\&A, 42, 685 


\section{Appendix A: Ground-based parallaxes}

We list the dates corresponding to the night of observations for the eight targets observed with IO:I on the $2 \mathrm{~m}$ robotic Liverpool telescope.

For each target with ground-based parallaxes derived from our Liverpool programme, we show two plots. On the left-hand side panels, we plot the residuals in right ascension (bottom) and declination (top), respectively. On the right-hand side panels, we display the relative positions in $\mathrm{X}$ and $\mathrm{Y}$ with the most precise fit to derive trigonomatric parallax.

Table A.1. Epochs of observations with IO:I on the Liverpool telescope for Hya02.

\begin{tabular}{ccc}
\hline \hline Name & Semester & $\begin{array}{c}\text { Epoch } \\
\text { yyymmdd }\end{array}$ \\
\hline Hya02 & CL15B06 & 20150827 \\
Hya02 & CL15B06 & 20150908 \\
Hya02 & CL15B06 & 20151111 \\
Hya02 & CL15B06 & 20160103 \\
Hya02 & CL15B06 & 20160214 \\
Hya02 & CL16B03 & 20160816 \\
Hya02 & CL16B03 & 20160903 \\
Hya02 & CL16B03 & 20160915 \\
Hya02 & CL16B03 & 20161015 \\
Hya02 & CL17B01 & 20171017 \\
Hya02 & CL17B01 & 20180114 \\
\hline
\end{tabular}

Table A.2. Epochs of observations with IO:I on the Liverpool telescope for Hya10.

\begin{tabular}{ccc}
\hline \hline Name & Semester & $\begin{array}{c}\text { Epoch } \\
\text { yyyymmdd }\end{array}$ \\
\hline Hya10 & CL15B06 & 20150904 \\
Hya10 & CL15B06 & 20150920 \\
Hya10 & CL15B06 & 20151005 \\
Hya10 & CL15B06 & 2015111 \\
Hya10 & CL15B06 & 20160102 \\
Hya10 & CL15B06 & 20160212 \\
Hya10 & CL16B03 & 20160825 \\
Hya10 & CL16B03 & 20160911 \\
Hya10 & CL16B03 & 20160925 \\
Hya10 & CL16B03 & 20161015 \\
\hline
\end{tabular}

Table A.3. Epochs of observations with IO:I on the Liverpool telescope for Hya09.

\begin{tabular}{ccc}
\hline \hline Name & Semester & $\begin{array}{c}\text { Epoch } \\
\text { yyyymmdd }\end{array}$ \\
\hline Hya19 & CL15B06 & 20150903 \\
Hya19 & CL15B06 & 20150928 \\
Hya19 & CL15B06 & 20151005 \\
Hya19 & CL15B06 & 20151006 \\
Hya19 & CL15B06 & 20151120 \\
Hya19 & CL15B06 & 20160101 \\
Hya19 & CL15B06 & 20160117 \\
Hya19 & CL15B06 & 20160209 \\
Hya19 & CL16B03 & 20160911 \\
Hya19 & CL16B03 & 20160924 \\
Hya19 & CL16B03 & 20161007 \\
Hya19 & CL16B03 & 20161022 \\
Hya19 & CL17B01 & 20171017 \\
Hya19 & CL17B01 & 20180103 \\
Hya19 & CL17B01 & 20180125 \\
\hline
\end{tabular}

Table A.4. Epochs of observations with IO:I on the Liverpool telescope for Hya11.

\begin{tabular}{ccc}
\hline \hline Name & Semester & $\begin{array}{c}\text { Epoch } \\
\text { yyyymmdd }\end{array}$ \\
\hline Hya11 & CL15B06 & 20150906 \\
Hya11 & CL15B06 & 20150920 \\
Hya11 & CL15B06 & 20151114 \\
Hya11 & CL15B06 & 20160103 \\
Hya11 & CL16B03 & 20160826 \\
Hya11 & CL16B03 & 20160911 \\
Hya11 & CL16B03 & 20160925 \\
Hya11 & CL16B03 & 20161015 \\
Hya11 & CL17B01 & 20171019 \\
Hya11 & CL17B01 & 20180119 \\
\hline
\end{tabular}


N. Lodieu et al.: A 3D view of the Hyades stellar and sub-stellar population

Table A.5. Epochs of observations with IO:I on the Liverpool telescope for Hya12.

\begin{tabular}{ccc}
\hline \hline Name & Semester & $\begin{array}{c}\text { Epoch } \\
\text { yyyymmdd }\end{array}$ \\
\hline Hya12 & CL15B06 & 20150906 \\
Hya12 & CL15B06 & 20150927 \\
Hya12 & CL15B06 & 20150928 \\
Hya12 & CL15B06 & 20151012 \\
Hya12 & CL15B06 & 20151120 \\
Hya12 & CL15B06 & 20160102 \\
Hya12 & CL15B06 & 20160118 \\
Hya12 & CL15B06 & 20160210 \\
Hya12 & CL16B03 & 20160817 \\
Hya12 & CL16B03 & 20160903 \\
Hya12 & CL16B03 & 20160918 \\
Hya12 & CL16B03 & 20161020 \\
Hya12 & CL16B03 & 20161022 \\
Hya12 & CL17B01 & 20171011 \\
Hya12 & CL17B01 & 20171018 \\
Hya12 & CL17B01 & 20180104 \\
Hya12 & CL17B01 & 20180123 \\
\hline
\end{tabular}

Table A.6. Epochs of observations with IO:I on the Liverpool telescope for HyaL5.

\begin{tabular}{ccc}
\hline \hline Name & Semester & $\begin{array}{c}\text { Epoch } \\
\text { yyyymmdd }\end{array}$ \\
\hline HyaL5 & CL15B06 & 20150906 \\
HyaL5 & CL15B06 & 20150921 \\
HyaL5 & CL15B06 & 20151007 \\
HyaL5 & CL15B06 & 20151120 \\
HyaL5 & CL15B06 & 20151218 \\
HyaL5 & CL15B06 & 20160209 \\
HyaL5 & CL16B03 & 20160905 \\
HyaL5 & CL16B03 & 20160923 \\
HyaL5 & CL16B03 & 20161008 \\
HyaL5 & CL16B03 & 20161015 \\
HyaL5 & CL17B01 & 20171018 \\
HyaL5 & CL17B01 & 20180113 \\
\hline
\end{tabular}

Table A.7. Epochs of observations with IO:I on the Liverpool telescope for CFHT-Hy-20.

\begin{tabular}{ccc}
\hline \hline Name & Semester & $\begin{array}{c}\text { Epoch } \\
\text { yyyymmdd }\end{array}$ \\
\hline CFHT-Hy-20 & CL15B06 & 20150907 \\
CFHT-Hy-20 & CL15B06 & 20150926 \\
CFHT-Hy-20 & CL15B06 & 20151013 \\
CFHT-Hy-20 & CL15B06 & 20151120 \\
CFHT-Hy-20 & CL15B06 & 20160102 \\
CFHT-Hy-20 & CL15B06 & 20160118 \\
CFHT-Hy-20 & CL15B06 & 20160207 \\
CFHT-Hy-20 & CL16B03 & 20160905 \\
CFHT-Hy-20 & CL16B03 & 20160922 \\
CFHT-Hy-20 & CL16B03 & 20161007 \\
CFHT-Hy-20 & CL16B03 & 20161022 \\
CFHT-Hy-20 & CL17B01 & 20171017 \\
CFHT-Hy-20 & CL17B01 & 20180119 \\
\hline
\end{tabular}

Table A.8. Epochs of observations with IO:I on the Liverpool telescope for CFHT-Hy-21.

\begin{tabular}{ccc}
\hline \hline Name & Semester & $\begin{array}{c}\text { Epoch } \\
\text { yyyymmdd }\end{array}$ \\
\hline CFHT-Hy-21 & CL15B06 & 20150906 \\
CFHT-Hy-21 & CL15B06 & 20150926 \\
CFHT-Hy-21 & CL15B06 & 20151012 \\
CFHT-Hy-21 & CL15B06 & 20151218 \\
CFHT-Hy-21 & CL16B03 & 20160818 \\
CFHT-Hy-21 & CL16B03 & 20160903 \\
CFHT-Hy-21 & CL16B03 & 20160918 \\
CFHT-Hy-21 & CL16B03 & 20161021 \\
CFHT-Hy-21 & CL17B01 & 20171018 \\
CFHT-Hy-21 & CL17B01 & 20180122 \\
\hline
\end{tabular}


A\&A 623, A35 (2019)
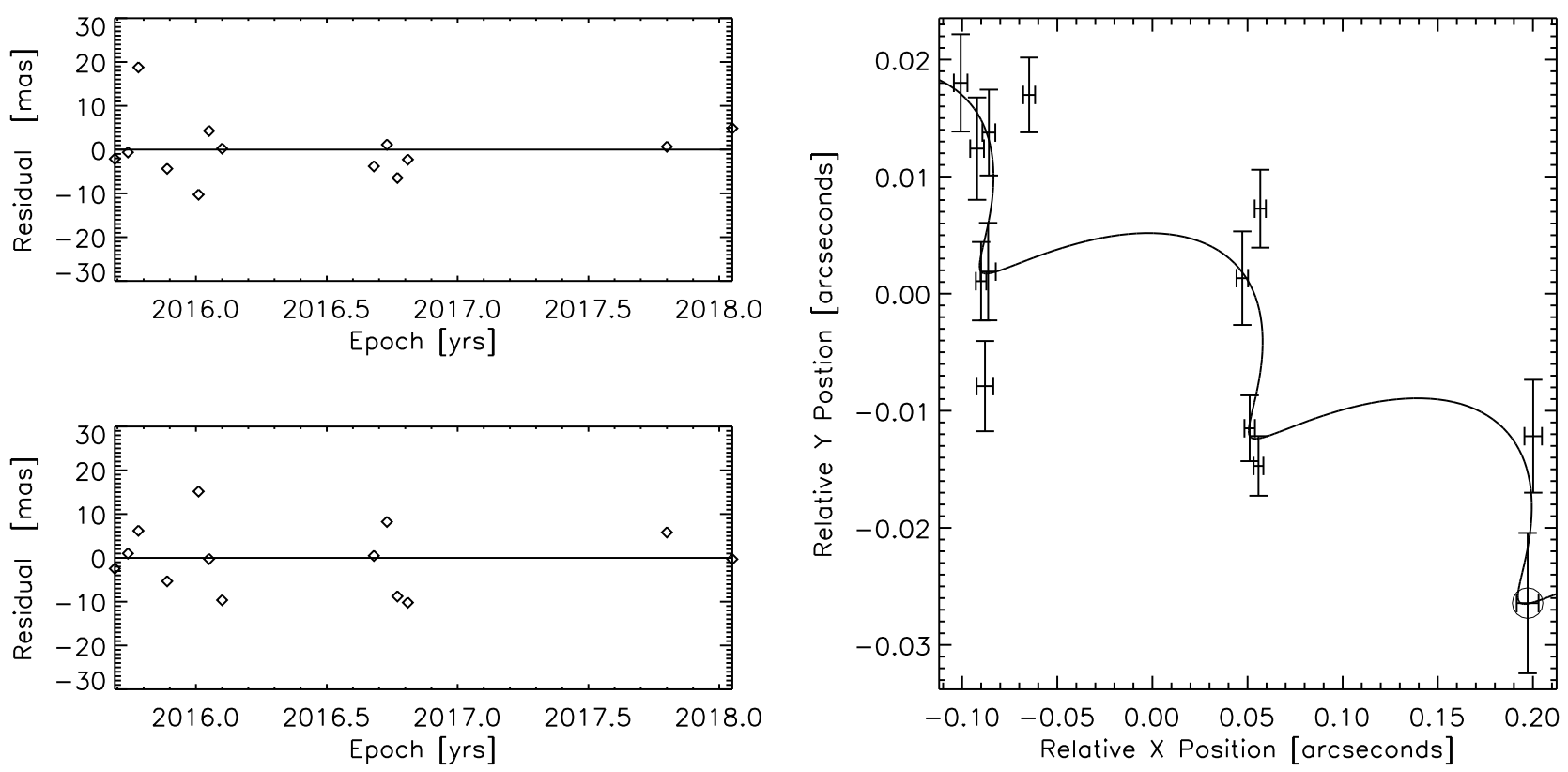

Fig. A.1. Left panels: residuals in mas as a function of epoch for right ascension (bottom) and declination (top). Right panel: solution for the parallax determination. We show the results for CFHT-Hy-20, the results for CFHT-Hy-21 are displayed in Fig. 3. The circle dot marks the reference epoch. 
N. Lodieu et al.: A 3D view of the Hyades stellar and sub-stellar population
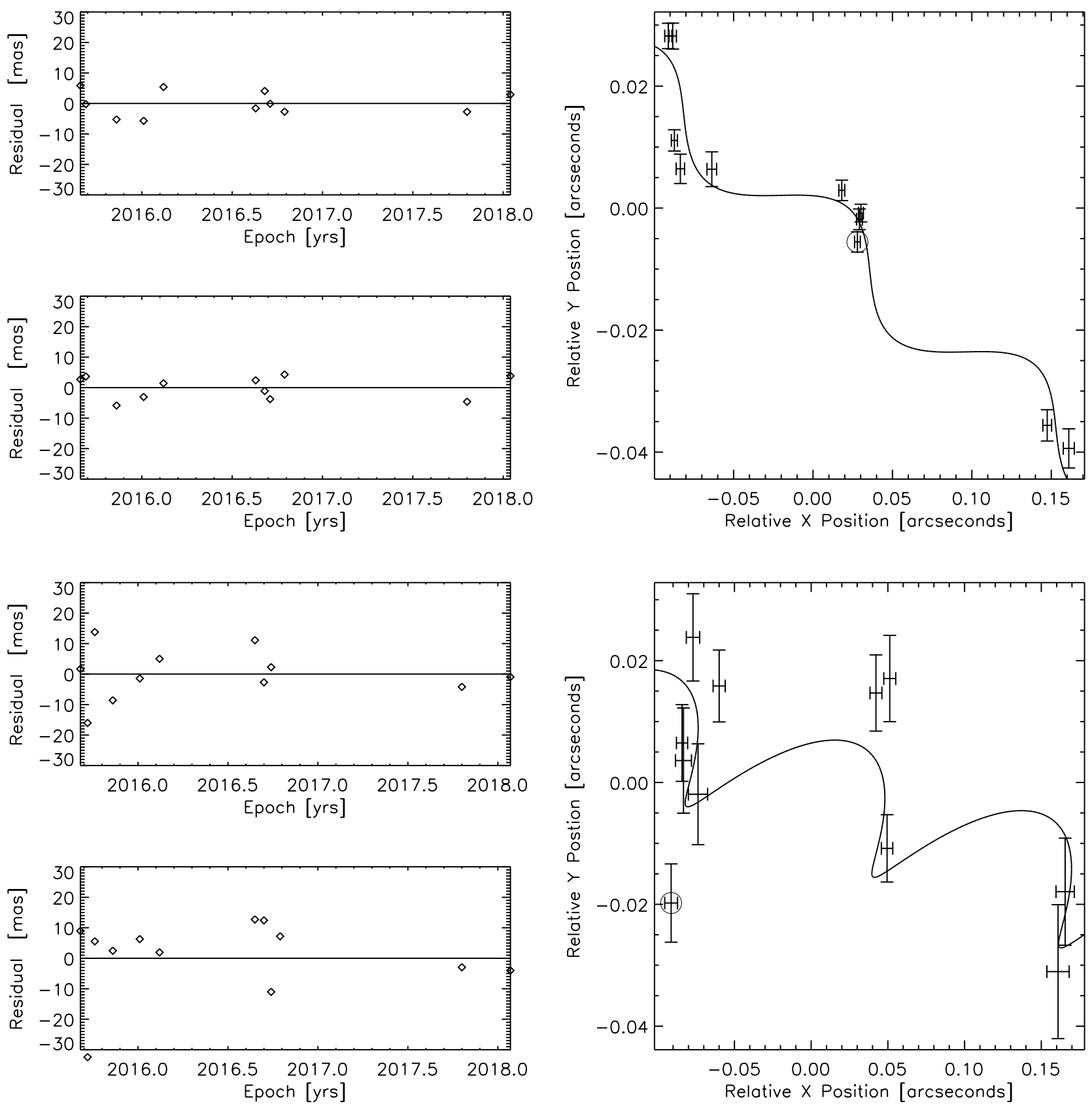

Fig. A.2. Same as Fig. A.1 but for Hya02 and Hya10. 
A\&A 623, A35 (2019)
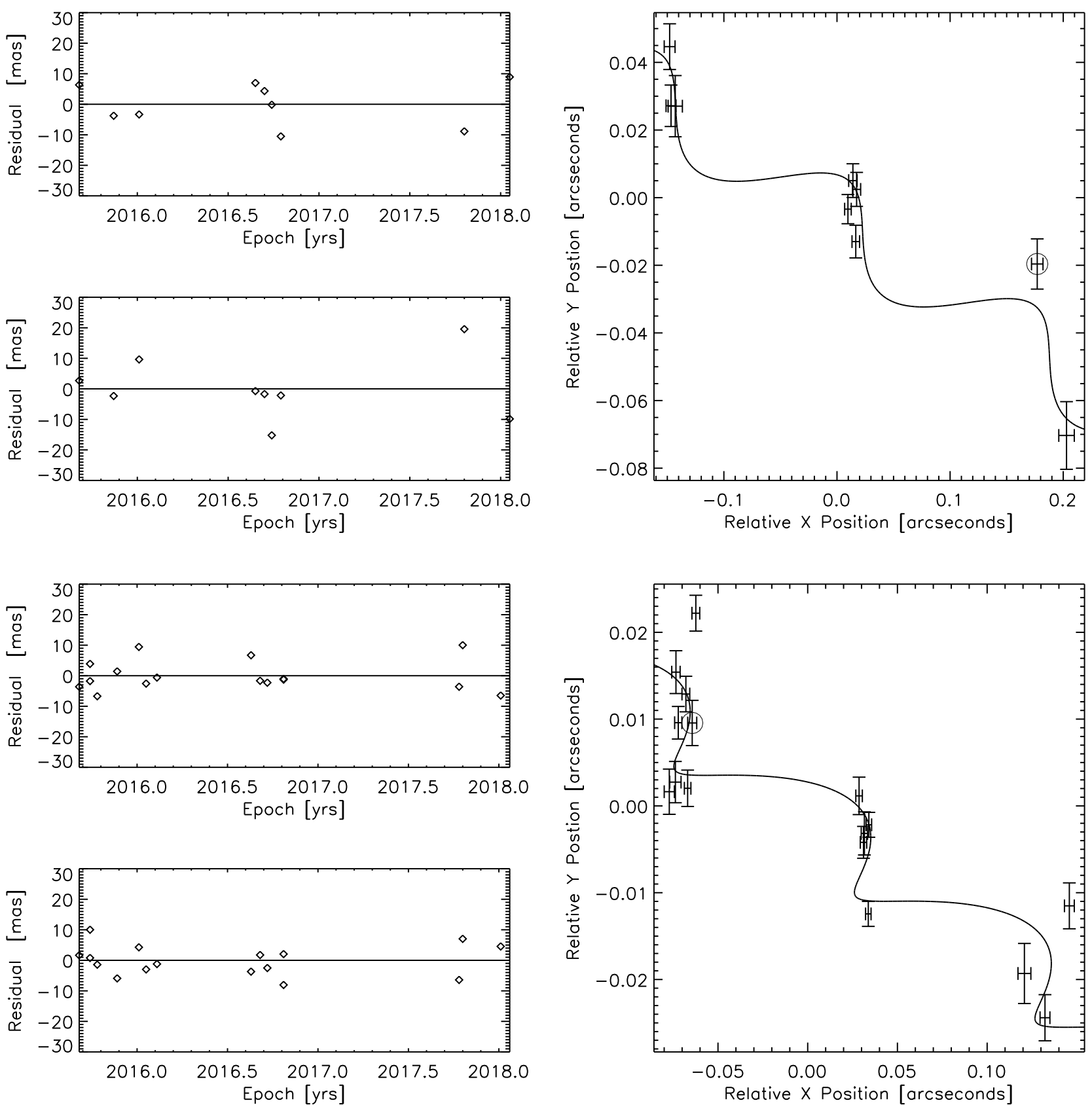

Fig. A.3. Same as Fig. A.1 but for Hya11 and Hya12. 
N. Lodieu et al.: A 3D view of the Hyades stellar and sub-stellar population
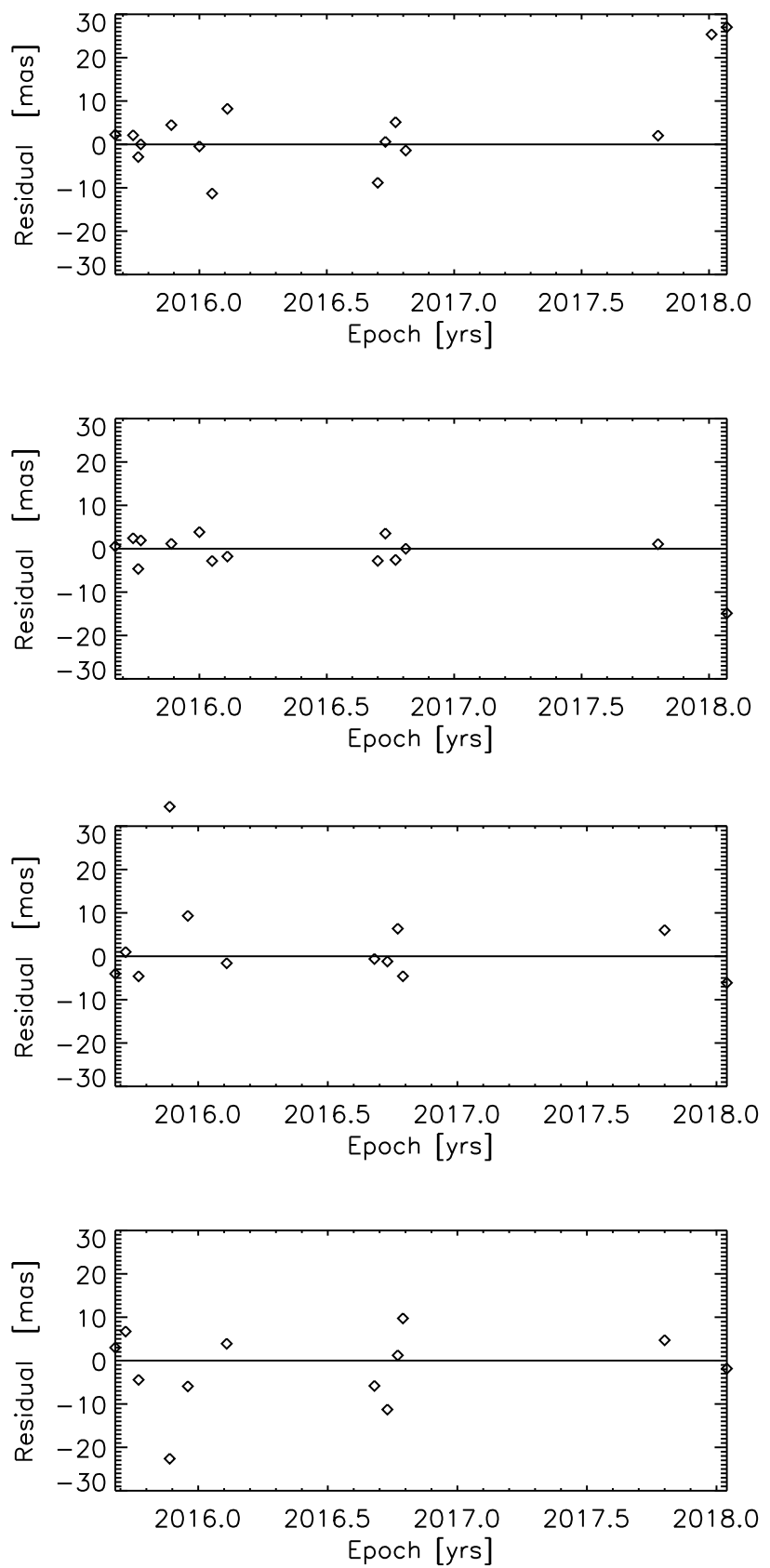

Fig. A.4. Same as Fig. A.1 but for Hya19 and HyaL5.
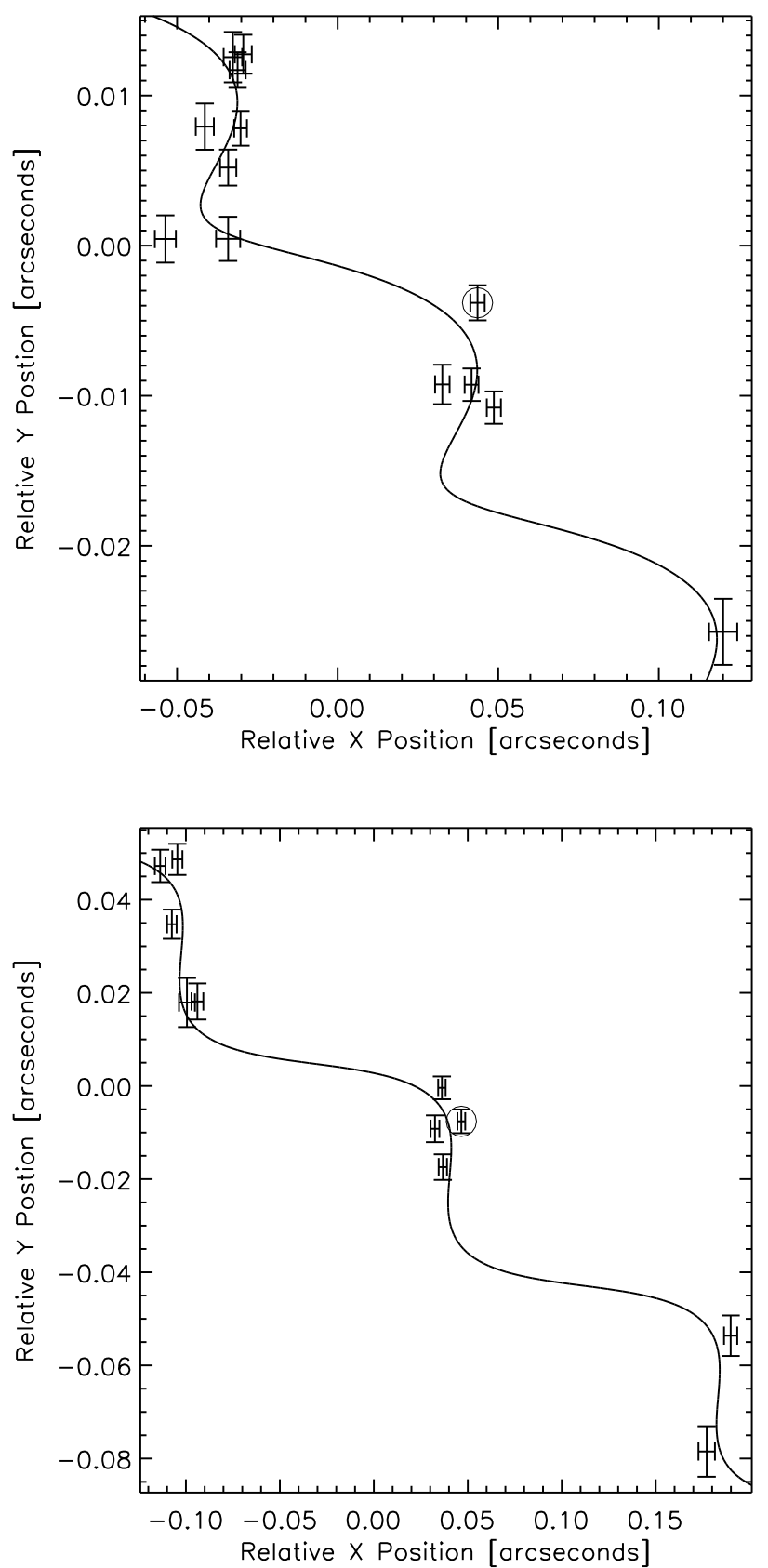


\section{Appendix B: The colour-magnitude diagrams}
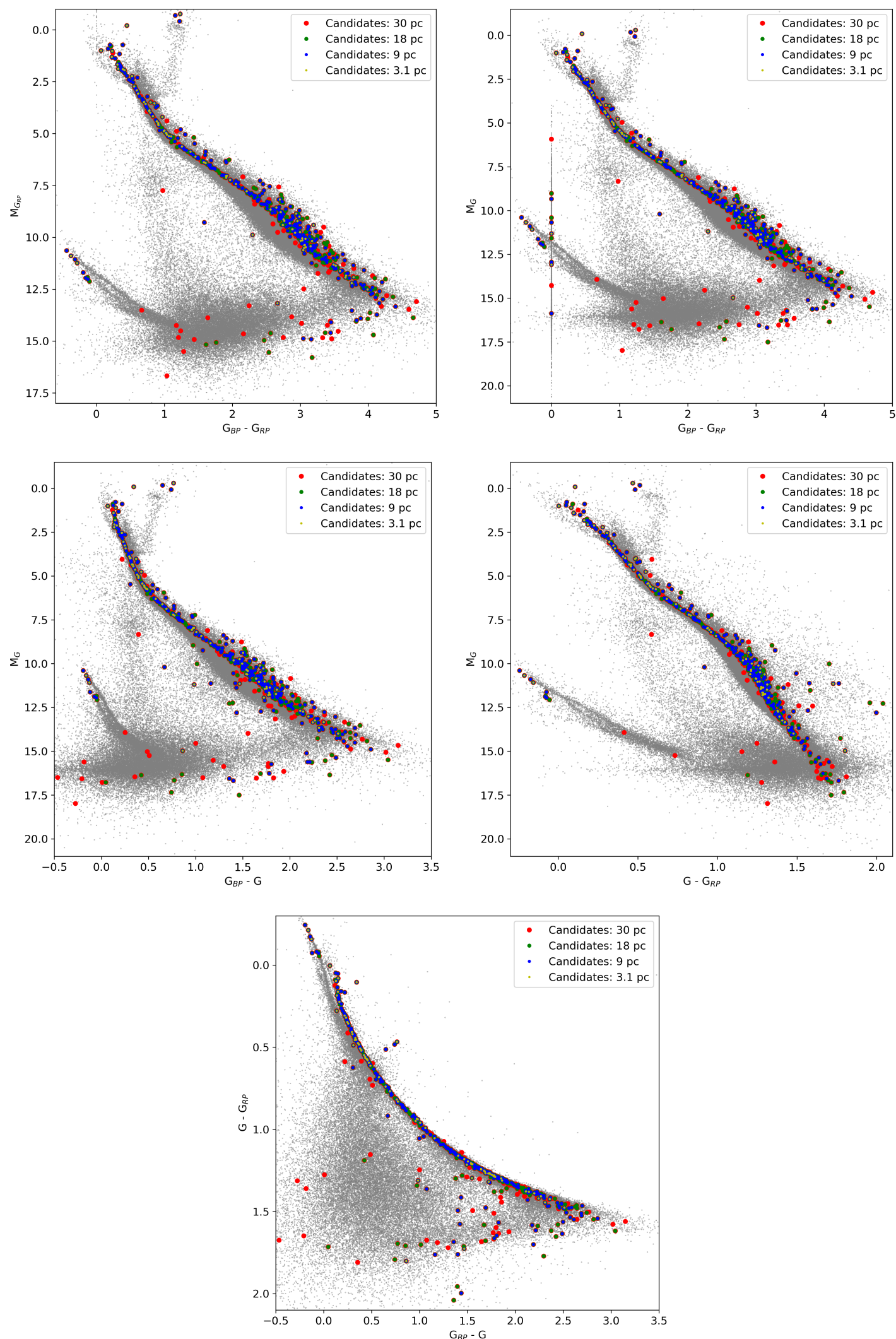

Fig. B.1. Colour-magnitude diagrams with Gaia photometry only for all candidates within a radius up to $30 \mathrm{pc}$ from the cluster centre. All candidates identified by the kinematic method are plotted in black. Members within 3.1,9,18, and $30 \mathrm{pc}$ are highlighted in yellow, green, blue, and red, respectively. Bottom panels: colour-colour diagram. We added as small grey dots the full Gaia catalogue over the $70^{\circ}$ radius. 
N. Lodieu et al.: A 3D view of the Hyades stellar and sub-stellar population
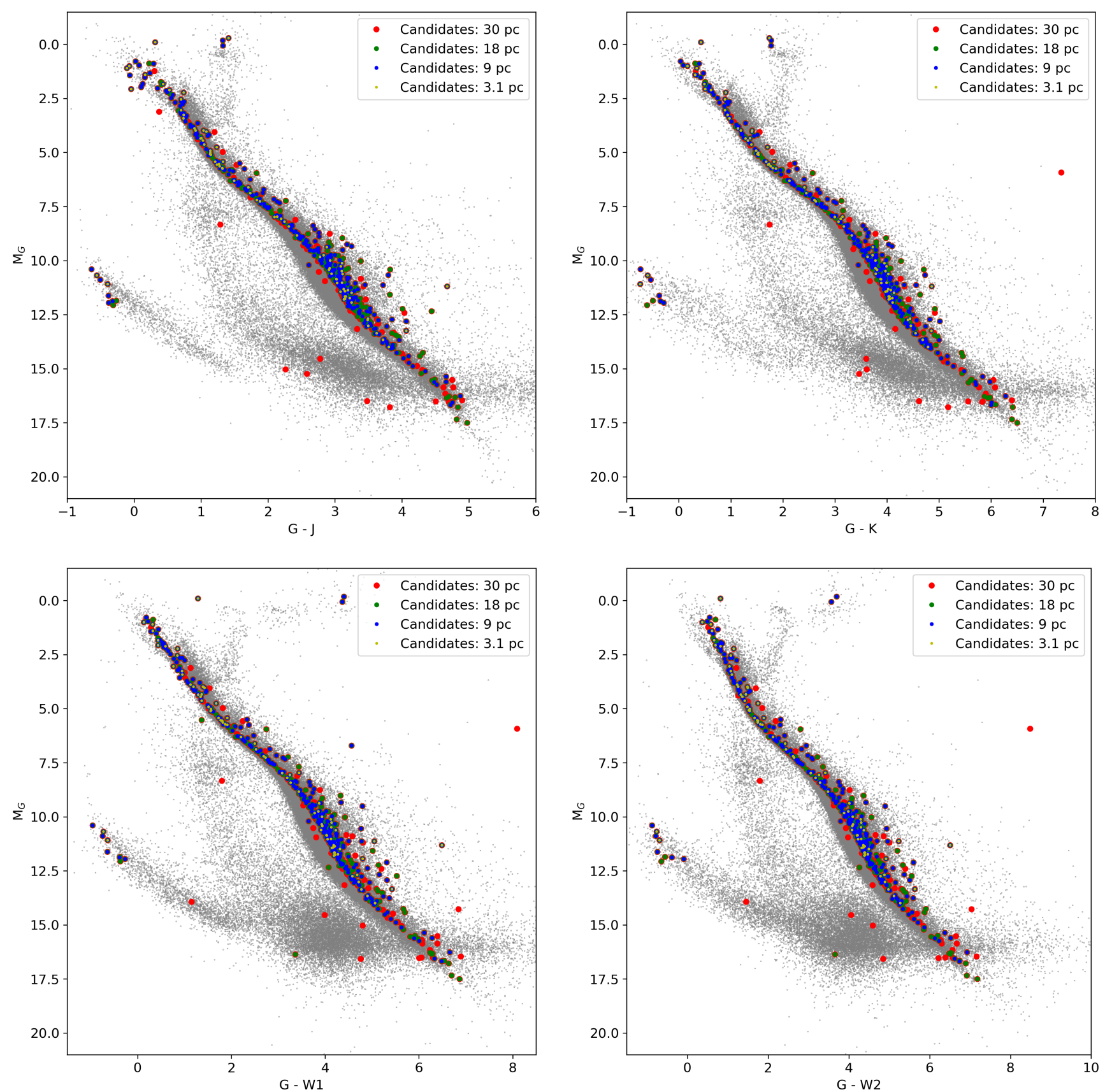

Fig. B.2. Colour-magnitude diagrams combining the Gaia magnitude with infrared photometry from $2 \mathrm{MASS}\left(J+K_{s}\right)$ and AllWISE $(W 1+W 2)$. Symbols are as in Fig. B.1. 

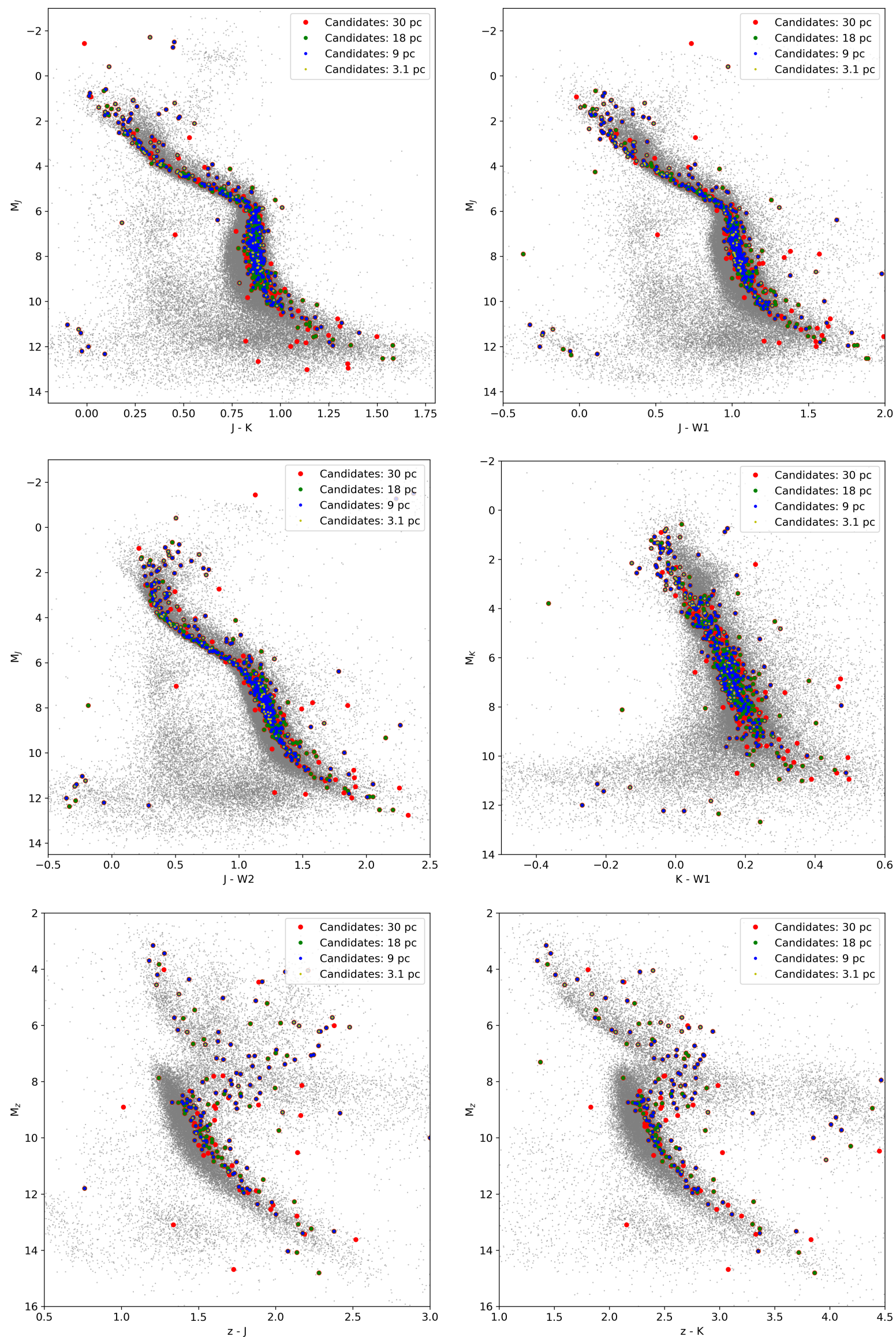

Fig. B.3. Colour-magnitude diagrams with non-Gaia photometric passbands. Symbols are as in Fig. B.1. Near-infrared filters are from 2MASS, optical ones from SDSS, and mid-infrared data from AllWISE. 


\section{Appendix C: Catalogues}

We plan to make public via CDS/Vizier the full table of all candidates within $70^{\circ}$ from the cluster centreafter applying the kinematic analysis described in Sect. 5.1. The full catalogue contains 1764 sources with Gaia DR2 data and photometry from several large-scale survey $(87,391,574,709$ sources within $3.1,9$, 18 , and $30 \mathrm{pc}$ from the cluster centre, respectively). We expect a much lower membership probability for sources beyond the $30 \mathrm{pc}$ radius because of the size of the cluster. Below we show a sub-set with some limited Gaia properties for space reasons, including source identifier, coordinates, proper motion, parallax, $G$ magnitude, galactic coordinates $\left(b_{x}, b_{y}, b_{z}\right)$, distance from the centreof the cluster, "c" parameter, mass (in $M_{\odot}$ ), and radial velocity when available. The full table will include photometry for all candidates from the large-scale surveys discussed in Sect. 2.

Table C.1. Catalogue of all 1764 candidates with a velocity consistent with the mean motion of the Hyades and located within a radius of $70^{\circ}$ from the cluster centre.

\begin{tabular}{|c|c|c|c|c|c|c|c|c|c|c|c|c|c|}
\hline SourceID & $\begin{array}{l}\text { RA } \\
\left({ }^{\circ}\right) \\
\end{array}$ & $\begin{array}{l}\text { Dec } \\
\left({ }^{\circ}\right)\end{array}$ & $\begin{array}{c}\text { pmRA } \\
\left(\operatorname{mas~yr}^{-1}\right)\end{array}$ & $\begin{array}{c}\text { pmDEC } \\
\left(\text { mas yr }^{-1}\right) \\
\end{array}$ & $\begin{array}{c}\text { Plx } \\
\text { (mas) }\end{array}$ & $\begin{array}{c}G \\
(\mathrm{mag})\end{array}$ & $\begin{array}{c}b_{x} \\
(\mathrm{pc})\end{array}$ & $\begin{array}{c}b_{y} \\
(\mathrm{pc})\end{array}$ & $\begin{array}{c}b_{z} \\
(\mathrm{pc})\end{array}$ & $\begin{array}{c}\text { d_centre } \\
(\mathrm{pc})\end{array}$ & $\mathrm{c}$ & $\begin{array}{c}\text { Mass } \\
M_{\odot} \\
\end{array}$ & $\begin{array}{c}\mathrm{RV} \\
\mathrm{km} \mathrm{s}^{-1}\end{array}$ \\
\hline 8479094371605632 & 45.013141699523 & 7.749846304858 & 327.9028 & 21.2424 & 42.0359 & 7.7084 & -16.998 & 3.208 & -16.270 & 27.674 & 8.713 & 0.8681 & 28.6366 \\
\hline 9167762312363520 & 51.251422487452 & 5.617677720416 & 65.0463 & 7.0610 & 11.1186 & 10.0217 & -68.895 & 3.234 & -58.926 & 48.526 & 8.966 & 0.9585 & $\mathrm{NaN}$ \\
\hline 11037726649058432 & 50.122631515919 & 8.454476681948 & 226.3237 & 6.1778 & 31.4749 & 9.2082 & -25.300 & 2.814 & -20.846 & 19.735 & 0.643 & 0.7525 & 31.0794 \\
\hline 13258873280731520 & 52.864927234039 & 10.658986726679 & 84.8280 & -0.0958 & 13.0153 & 19.7575 & -62.054 & 6.372 & -45.067 & 33.605 & 3.285 & 0.0824 & $\mathrm{NaN}$ \\
\hline 16744020197785216 & 51.277548426737 & 12.471543437172 & 74.9678 & -7.0012 & 10.1848 & 19.2007 & -78.887 & 12.201 & -57.187 & 54.176 & 16.177 & 0.0963 & $\mathrm{NaN}$ \\
\hline 26259120810103552 & 37.238361188313 & 12.003498952161 & 85.1459 & -2.3126 & 10.2194 & 7.9276 & -64.764 & 27.150 & -68.465 & 61.461 & 18.824 & 1.4359 & 23.5105 \\
\hline 27821118811895808 & 46.563573756827 & 12.098487746919 & 225.1146 & -5.2259 & 29.6763 & 14.6019 & -25.610 & 5.838 & -21.218 & 20.142 & 2.288 & 0.1704 & $\mathrm{NaN}$ \\
\hline 29720761371785216 & 47.681557651709 & 13.643193800393 & 155.6586 & -6.3771 & 20.9761 & 12.5629 & -37.463 & 8.664 & -29.023 & 16.241 & 0.862 & 0.4792 & 30.6009 \\
\hline 34762198279394048 & 46.412355333009 & 16.976051403957 & 179.1221 & -14.2885 & 23.9412 & 16.4948 & -32.859 & 9.900 & -24.197 & 16.744 & 2.957 & 0.1141 & $\mathrm{NaN}$ \\
\hline .. & $\ldots$ & $\ldots$ & $\ldots$ & $\ldots$ & $\ldots$ & $\because$ & $\cdots$ & $\ldots$ & $\ldots$ & $\ldots$ & .. & $\cdots$ & $\cdots$ \\
\hline 5717193115607283200 & 115.422143085005 & -17.887725569764 & -1.1807 & -0.4641 & 11.0735 & 20.9923 & -52.535 & -73.735 & 4.002 & 77.284 & 6.730 & 0.0731 & $\mathrm{NaN}$ \\
\hline 5718970132565285760 & 116.242950895585 & -16.193647715707 & -31.7571 & 44.5291 & 12.1243 & 19.5637 & -49.117 & -66.247 & 5.842 & 70.425 & 6.867 & 0.0864 & $\mathrm{NaN}$ \\
\hline 5725289644362539008 & 119.044895757239 & -15.184292651653 & -34.3797 & 31.8224 & 11.5440 & 15.2876 & -52.342 & -71.862 & 10.745 & 77.629 & 17.000 & 0.2955 & $\mathrm{NaN}$ \\
\hline 5725439860839859968 & 119.858101150168 & -14.393897858044 & -17.9755 & 21.9587 & 11.0164 & 20.3713 & -53.256 & -72.369 & 12.581 & 78.869 & 13.110 & 0.0796 & $\mathrm{NaN}$ \\
\hline 5725579537475527168 & 118.457254215531 & -14.447942614473 & -21.1209 & 22.7763 & 12.9785 & 20.9897 & -46.103 & -61.187 & 9.101 & 66.740 & 12.216 & 0.0705 & $\mathrm{NaN}$ \\
\hline 5751202552146851328 & 126.536913865475 & -11.386095441641 & -29.6120 & 23.9180 & 10.1213 & 19.6470 & -55.898 & -78.419 & 25.968 & 90.342 & 20.502 & 0.0899 & $\mathrm{NaN}$ \\
\hline 5753630656073435904 & 128.709626532580 & -8.927219398629 & -60.4998 & 30.3963 & 12.4754 & 17.7951 & -45.331 & -61.377 & 25.110 & 74.637 & 0.577 & 0.1174 & $\mathrm{NaN}$ \\
\hline 5761914926433163648 & 132.322528914351 & -3.812445055310 & -58.3341 & 20.8782 & 12.4201 & 16.8128 & -46.366 & -57.202 & 32.757 & 76.032 & 14.000 & 0.1529 & $\mathrm{NaN}$ \\
\hline 5763795087020644224 & 132.758229193570 & -2.549832351491 & -78.5643 & 26.5395 & 18.8073 & 19.3266 & -30.978 & -36.978 & 22.513 & 55.963 & 21.976 & 0.0784 & $\mathrm{NaN}$ \\
\hline
\end{tabular}

Notes. The full catalogue is available at the CDS. 